\title{
Data Collection for the Fourth Multicentre Confidential Enquiry into Perioperative Equine Fatalities (CEPEF4) Study: New Technology and Preliminary Results
}

\author{
Miguel Gozalo-Marcilla ${ }^{1, *} \mathbb{(}$, Regula Bettschart-Wolfensberger ${ }^{2}\left(\mathbb{D}\right.$, Mark Johnston ${ }^{3}$, Polly M. Taylor ${ }^{4}(\mathbb{D}$ \\ and Jose I. Redondo ${ }^{5}$ (D) \\ 1 Veterinary Clinical Sciences, The Royal (Dick) School of Veterinary Studies and The Roslin Institute, \\ Easter Bush Campus, The University of Edinburgh, Edinburgh EH25 9RG, UK \\ 2 Department of Clinical Diagnostics and Services, Vetsuisse Faculty, University of Zürich, \\ 8057 Zürich, Switzerland; rbettschart@vetclinics.uzh.ch \\ 3 Vetstream Ltd., Three Hills Farm, Bartlow, Cambridge CB21 EN, UK; mark.johnston@vetstream.com \\ 4 Taylor Monroe, Little Downham, Cambridge CB6 2TY, UK; polly@taylormonroe.co.uk \\ 5 Departamento de Medicina y Cirugía Animal, Facultad de Veterinaria, Universidad Cardenal Herrera-CEU, \\ CEU Universities, 46115 Valencia, Spain; nacho@uchceu.es \\ * Correspondence: miguelgozalomarcilla@gmail.com; Tel.: +44-(0)-131-651-7366
}

\section{check for}

updates

Citation: Gozalo-Marcilla, M.; Bettschart-Wolfensberger, R.;

Johnston, M.; Taylor, P.M.; Redondo,

J.I. Data Collection for the Fourth

Multicentre Confidential Enquiry into

Perioperative Equine Fatalities

(CEPEF4) Study: New Technology

and Preliminary Results. Animals

2021, 11, 2549. https://doi.org/

10.3390/ani11092549

Academic Editor: John A. E. Hubbell

Received: 5 July 2021

Accepted: 25 August 2021

Published: 30 August 2021

Publisher's Note: MDPI stays neutral with regard to jurisdictional claims in published maps and institutional affiliations.

Copyright: (c) 2021 by the authors. Licensee MDPI, Basel, Switzerland. This article is an open access article distributed under the terms and conditions of the Creative Commons Attribution (CC BY) license (https:/ / creativecommons.org/licenses/by/ $4.0 /)$.
Simple Summary: New technologies allow researchers to improve the methods for immediate, accurate data collection, cleaning and analysis, with minimal geographical limitations. Although much has improved in the field of equine anaesthesia in recent years, we are still far from reducing anaesthetic-related mortality in this species in comparison with small animal anaesthesia. The aim of this multicentre study was to probe the usefulness of an internet-based method that utilised an electronic questionnaire and statistical software to show the data and report outcomes from horses undergoing general anaesthesia and certain procedures using standing sedation. Within six months, 8656 cases from 69 centres were collected: 6701 procedures under general anaesthesia and 1955 under standing sedation. The results demonstrated (i) the utility of the method and (ii) that some horses died unexpectedly when undergoing not only general anaesthesia, but also standing sedation. Finally, (iii) we present some descriptive data that outline the current anaesthesia practice compared with the previous CEPEF2. We concluded that our internet-based method is suitable for this type of study. New techniques may reduce the mortality rate. However, the results presented here should be interpreted cautiously as these are only preliminary data with lower numbers than CEPEF2.

Abstract: It is almost 20 years since the largest observational, multicentre study evaluating the risks of mortality associated with general anaesthesia in horses. We proposed an internet-based method to collect data (cleaned and analysed with R) in a multicentre, cohort, observational, analytical, longitudinal and prospective study to evaluate peri-operative equine mortality. The objective was to report the usefulness of the method, illustrated with the preliminary data, including outcomes for horses seven days after undergoing general anaesthesia and certain procedures using standing sedation. Within six months, data from 6701 procedures under general anaesthesia and 1955 standing sedations from 69 centres were collected. The results showed (i) the utility of the method; also, that (ii) the overall mortality rate for general anaesthesia within the seven-day outcome period was $1.0 \%$. In horses undergoing procedures other than exploratory laparotomy for colic ("noncolics"), the rate was lower, $0.6 \%$, and in "colics" it was higher, at $3.4 \%$. For standing sedations, the overall mortality rate was $0.2 \%$. Finally, (iii) we present some descriptive data that demonstrate new developments since the previous CEPEF2. In conclusion, horses clearly still die unexpectedly when undergoing procedures under general anaesthesia or standing sedation. Our method is suitable for case collection for future studies.

Keywords: anaesthesia; CEPEF; data analysis; death; epidemiology; equine; horse; mortality; standing sedation 


\section{Introduction}

Internet-based research has become commonplace during the last 10-20 years and makes online data collection possible from a large pool of participants with few geographical limitations. Online data collection is quick, cheap and increases the accuracy and efficacy of data entry [1]. Moreover, data can be analysed interactively with the ability to follow-up with participants [1-3]. Apart from understanding how to use new technology and the expertise required to match the study design with data collection, adequate statistical software for data analysis and cleaning is mandatory [4,5]. Immediate technical support and fluent communication between the administrators and participants is also crucial.

The high risk of mortality associated with general anaesthesia in horses remains one of the biggest concerns for equine practitioners and veterinary anaesthetists. Many studies report anaesthesia-related mortality risks. Most of these studies are retrospective [6-14] but a few prospective single-centre [15] and some prospective, multi-centre $[16,17]$ investigations have been carried out. To date, the Confidential Enquiry into Perioperative Equine Fatalities 2 (CEPEF2) published in 2002 remains the largest observational, multicentre study with 41,824 cases collected from 62 clinics over a period of 6 years [18]. The overall death rate up to seven days was $1.9 \%, 0.9 \%$ in noncolics and $7.8 \%$ in colics [18]. Although much has changed since then, we are still far from reducing these numbers [19], and the need for an update on the CEPEF data was proclaimed eight years ago [20]. Avoiding general anaesthesia by undertaking some procedures in standing horses may reduce the mortality, but there are no data as yet to support this hypothesis.

The first objective of this report was to describe the usefulness of an internet-based method for data collection and a strategy for data cleaning for the CEPEF4 study, whose final aim is to identify the risk factors associated with equine anaesthesia and standing sedation. Second, to report the outcomes/fatalities within a period of seven days of equidae undergoing (i) general anaesthesia and (ii) certain procedures performed under standing sedation during the first six months of CEPEF4 and to describe the key preliminary findings that will be analysed in detail in the future. Our first hypothesis was that the proposed method would be a fast, reliable tool to collect, clean and analyse data. Second, that mortality was lower than reported by Johnston et al. almost 20 years ago [18] and that standing sedation procedures are not exempt from the risk of death, although it is reduced. Finally, we hypothesised that there would be trends towards new practices in anaesthesia and analgesia compared with the previous CEPEF2.

\section{Materials and Methods}

The study design was multicentre, cohort, observational, analytical, longitudinal and prospective. For this phase of preliminary results, the six-month data collection period was from 1 November 2020 until 30 April 2021.

Inclusion criteria comprised cases of horses, donkeys and mules of all ages from clinics all over the world, recruited specifically for this project. The equidae involved were all cases from each clinic undergoing (i) general anaesthesia whatever the reason and (ii) standing sedation for surgery or advanced diagnostic imaging (magnetic resonance imaging (MRI), computed tomography (CT) or scintigraphy) requiring a continuous rate infusion (CRI) or at least one extra top-up apart from the initial sedation bolus. Once recording started in any one clinic, all cases had to be included. However, to allow for holidays and staff absence, all cases within a specified period could be omitted; recording and sending all cases started again after the specified period.

Exclusion criteria were cases outside the agreed recording periods, cases from centres that did not follow the communication process and cases from centres that did not send all the cases from a recording period. General anaesthetics for terminal procedures were excluded, as well as standing sedations without top-ups or CRIs, and standing sedations for reasons other than surgery or advanced diagnostic imaging, such as cast changes or sinuscopies. 
The steps followed in this study are detailed below:

(1) Preparation of the questionnaire

A user-friendly, online questionnaire used in small animals [21] was adapted for this equine study using the feedback received from a group of researchers and clinicians with special interest in equine anaesthesia and analgesia [22]. It was designed to be used to compile information for both general anaesthesia and standing sedation.

Briefly, the questionnaire collected data about the centre, the level of training of the responsible anaesthetist, the patient, the procedure, the anaesthetic and analgesic protocols and other details about management of the anaesthesia or sedation. Information about the anaesthetic induction and recovery phases was included, with the potential to indicate any intraoperative complications and details of the postoperative period for up to seven days. The questionnaire and the instructions to complete it are available on a website created for support and promotion at https: / / cepef4.wordpress.com (accessed on 6 June 2021), and are provided here as Supplementary Materials S1.

(2) Definition of perioperative equine fatalities

The same classification of fatalities was followed as used in previous CEPEF studies $[16,18]$. After induction of general anaesthesia or the first sedation bolus for standing sedation, the outcome was recorded at day seven for each case as (i) alive (or discharged), (ii) put to sleep (PTS)/euthanised or (iii) dead. The time of PTS or dead was recorded as premedication, induction, maintenance, recovery or the day up to day seven. Each centre was encouraged to use an outcome logbook to communicate further which colic or noncolic cases were alive, PTS or dead during this seven-day period.

When a horse was euthanized due to an inoperable lesion found at surgery, due to pre-existing disease or financial constraints, the outcome was classified as PTS. When a horse died unexpectedly or was euthanised due to a perioperative complication, such as a fracture in recovery, the outcome was classified as death. Other examples of death include intraoperative cardiac arrest, spinal cord malacia requiring euthanasia during recovery or post-operative myopathy requiring euthanasia on humane grounds up to seven days after surgery. Those classified as noncolic deaths were required to complete a more detailed online survey at https://edinburgh.onlinesurveys.ac.uk/equine-ga-mortalityform (accessed on 6 June 2021).

Classification of outcomes was performed by M.G.-M. and J.I.R., and later confirmed by R.B.-W., M.J. and P.M.T. Ultimately, before final data analysis, the outcomes were confirmed with the contact person from each centre, referred to as the ambassador (see (10) in Methods).

(3) Ethics statement

The study was approved by the international Ethical Review Committee of the Association of Veterinary Anaesthetists (AVA), under protocol 2020-009 on 4 September 2020. Full details of the application form were provided to any centre that also required Local Ethical Committee Approval from their institution.

(4) Recruitment of collaborating centres

Recruitment included an abstract presented at the AVA Spring meeting in Dublin 2020 [23], and Correspondence to the Editor published in peer reviewed journals targeting equine practitioners [24] and veterinary anaesthetists [22], respectively. Finally, we also used our professional network and contacted several centres personally.

(5) The Ambassador figure and the Agreement Form

Ambassadors for each centre volunteered or were recruited to be the contact person who also took responsibility for the centre's data collection. 
Before sending the data, the ambassador signed an agreement form (https://cepef4 .wordpress.com/forms-instructions-and-help/ (accessed on 6 June 2021)) ensuring to cooperate/supervise selflessly, providing good quality data including all the cases within a period of time to avoid bias. The CEPEF team certified the anonymous handling of the data.

\section{(6) Anonymity and confidentiality of each patient, owner and centre}

As in previous CEPEF studies, the anonymity and confidentiality of the patients, owners and centres was ensured. Identification of each patient was provided by a number given by the centre for further communication with the CEPEF team. These numbers were used only for communication with each centre. Each case entered in the database received a unique CEPEF number for data handling and analysis. Moreover, each centre had a code known only by M.G.-M. and J.I.R. The remaining authors, R.B.-W., J.I.R. and P.M.T., were not aware of centre coding for further evaluation of the data.

(7) The communication process (recruitment and follow-up meetings)

We instituted a method of interactive communication with the ambassador of each centre always performed by the same investigator (M.G.-M.) in order to standardize the information and, therefore, reduce data inconsistencies. For each centre, the project was first presented to the ambassador, and ideally, also to the whole team involved in data collection, via an online meeting (Teams ${ }^{\circledR}$ (Microsoft Corporation, Redmond, WA, USA), Zoom ${ }^{\circledR}$ (Zoom Video Communications Inc., San Jose, CA, USA), Skype ${ }^{\circledR}$ (Microsoft Corporation, Redmond, WA, USA), Meet ${ }^{\circledR}$ (Google Inc., Mountain View, CA, USA)) of about one hour's duration. This included a standard presentation of the project, introduction to the study website and explained the questionnaire and its technicalities. At least a second, and ideally a third, follow-up meeting was subsequently organised to ensure complete familiarity with the system.

(8) Data collection and storage

The electronic questionnaire was used to collect the data (Supplementary Materials S1). A .pdf file was programmed using Adobe Acrobat Pro ${ }^{\circledR}$ (Adobe Inc., San Jose, CA, USA). This .pdf file can be completed using the free app Adobe Acrobat Reader DC (Adobe Inc., San José, CA, USA), available for the different operating systems (Android ${ }^{\circledR}$ (Google Inc., Mountain View, CA, USA), IOS ${ }^{\circledR}$ (Apple, Cupertino, CA, USA), Windows ${ }^{\circledR}$ (Microsoft Corporation, Redmond, WA, USA) and $\operatorname{macOS}^{\circledR}$ (Apple, Cupertino, CA, USA)). The questionnaire can be completed on any internet-connected device, including mobile phones, tablets, laptops or computers. Once completed, the questionnaire is sent by e-mail to a specific e-mail account in which all the questionnaires are stored. The collected metadata is converted to a .csv file that is added to the main database as a new case.

(9) Data cleaning and statistics

Data processing and statistical analyses were performed using R 4.1.0 [25]. Data quality was improved using an initial data cleaning phase performed with specific scripts searching for inconsistencies. R scripts were introduced to detect duplicated cases or blank fields as non-available data (-NA-) in the studied variables. Other R scripts detected more sophisticated inconsistencies using Boolean algebra, for instance, in general anaesthetics coded as inhalation anaesthesia only (INH) but where an alpha $a_{2}$-agonist CRI was ticked, or when an inhalant agent was ticked for a standing sedation procedure. In addition, further $\mathrm{R}$ scripts were used to detect uncommon anaesthetic practices, such as induction with an inhalant agent or when premedication was not administered. The full list of the R scripts used in this study can be found in the Supplementary Materials S2.

The ambassador of any centre where data inconsistencies were detected was contacted and sent an Excel ${ }^{\circledR}$ (Microsoft Corporation, Redmond, WA, USA) file by e-mail to confirm whether the inconsistencies were real and to request revision if so. These detected inconsistencies were corrected manually within the database, only with the strict agreement of the ambassador. 
Once data were cleaned, a descriptive analysis was performed. Tables and figures were created using the following R packages: table1 (v1.4.1) [26], ggplot2 (v3.3.3) [27] and rnaturalearth (v0.1.0) [28]. Variables that followed a normal distribution are shown as mean \pm standard deviation, whereas those non-normally distributed are shown as median [range].

(10) Final meeting with each ambassador to double-check data and outcomes

The individual database from each centre was sent to the ambassador of the centre before the final meeting. This included all the cases sent up until 30 April 2021. A personalized report for the centre was included for its approval. Data were always double-checked by the principal investigator (M.G.-M.) and the ambassador for each centre during a scheduled online meeting of about 30 to $90 \mathrm{~min}$, depending on the volume of the data and potential inconsistencies. The ambassadors were asked for feedback and to grade the project and the communication process between 0 (worse) to 10 (excellent). These meetings occurred between Monday 17 May and Thursday 3 June 2021.

Figure 1 shows the flow diagram of the process.

Phase 1. Initiation and planning

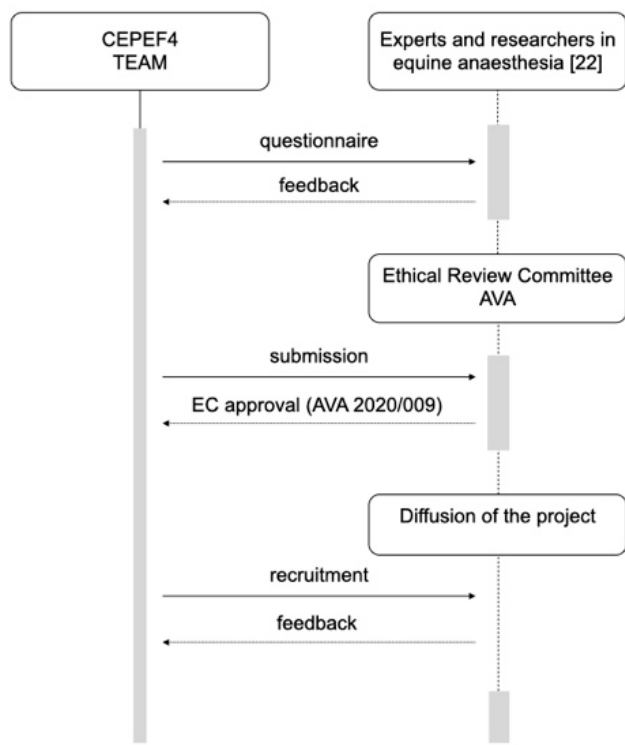

Phase 2. Execution, monitoring and controlling

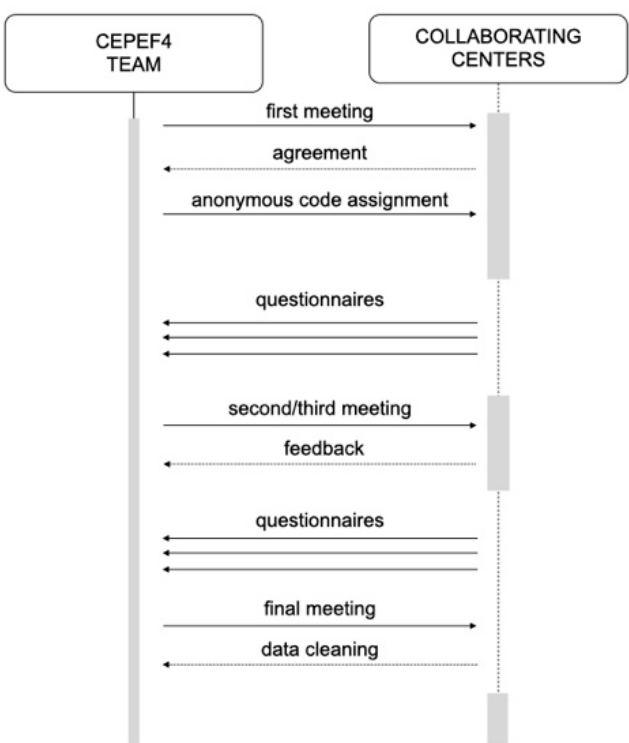

Figure 1. Flow diagram of the process.

(11) Reporting the results

The STROBE-Vet guidelines, an extension of the STROBE (Strengthening the Reporting of Observational Studies in Epidemiology) statement [29], as recommended for reporting of observational studies in veterinary medicine (https: / / strobevet-statement.org, accessed on 6 June 2021), (Supplementary Materials S3) were followed in order to maximise reporting quality. 


\section{Results}

During the specified first six months, 69 centres from 20 countries in four continents collaborated to collect data (Figure 2, Table 1). As a result of continuous recruitment, each centre had a different starting date for data collection.

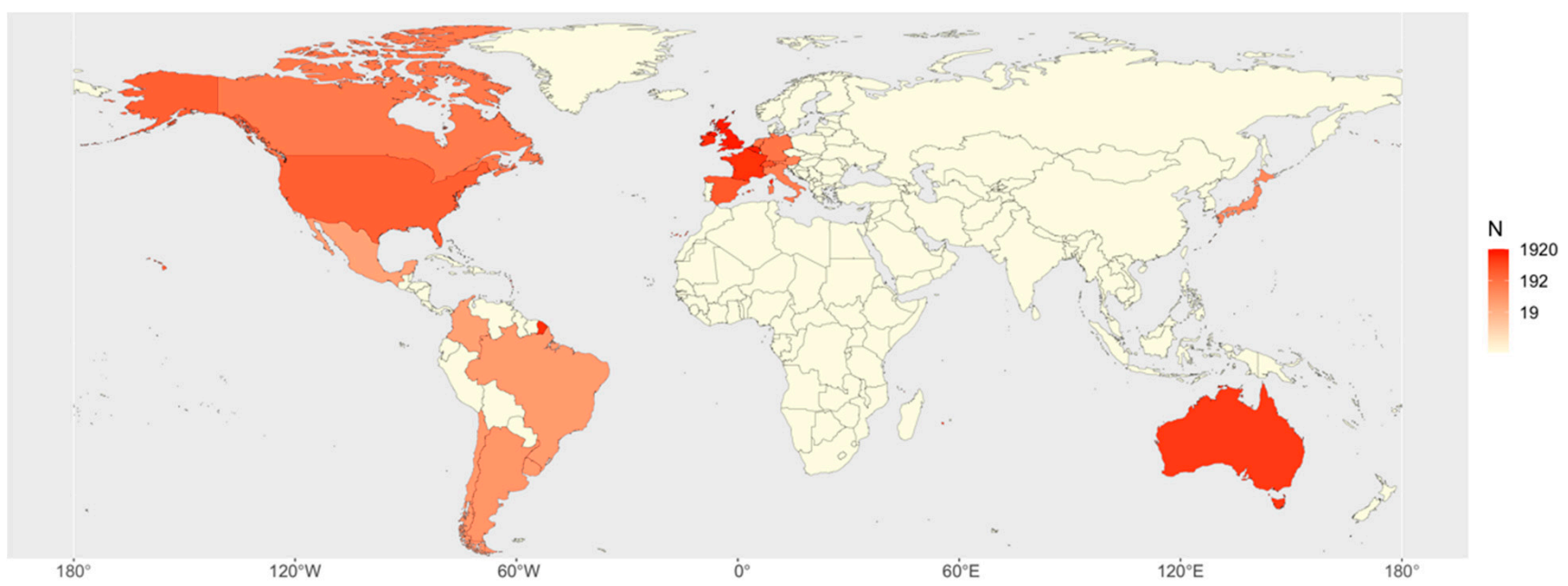

Figure 2. Heat map of the distribution of the cases by country.

Table 1. Countries of origin of the 69 collaborating centres classified per type of hospital/clinic and cases and percentage of cases registered for each country.

\begin{tabular}{|c|c|c|c|c|c|c|}
\hline Country & $\begin{array}{l}\text { University } \\
\text { Hospitals }\end{array}$ & $\begin{array}{c}\text { Referral } \\
\text { Centres }\end{array}$ & $\begin{array}{l}\text { Ambulatory } \\
\text { Clinicians }\end{array}$ & Total Centres & Cases $^{1}$ & $\%$ Cases \\
\hline Belgium & 2 & 2 & 0 & 4 & 1906 & 21.78 \\
\hline United Kingdom & 3 & 10 & 0 & 13 & 1670 & 19.08 \\
\hline Republic of Ireland & 1 & 2 & 0 & 3 & 1068 & 12.20 \\
\hline France & 0 & 6 & 0 & 6 & 1025 & 11.71 \\
\hline Australia & 0 & 2 & 0 & 2 & 898 & 10.26 \\
\hline Switzerland & 2 & 2 & 0 & 4 & 444 & 5.07 \\
\hline Spain & 6 & 2 & 0 & 8 & 399 & 4.56 \\
\hline United States of America & 3 & 0 & 0 & 3 & 327 & 3.74 \\
\hline Germany & 1 & 0 & 0 & 1 & 162 & 1.85 \\
\hline The Netherlands & 1 & 1 & 0 & 2 & 150 & 1.71 \\
\hline Canada & 2 & 0 & 0 & 2 & 142 & 1.62 \\
\hline Italy & 2 & 0 & 0 & 2 & 120 & 1.37 \\
\hline Austria & 1 & 0 & 0 & 1 & 97 & 1.11 \\
\hline Japan & 0 & 1 & 0 & 1 & 82 & 0.94 \\
\hline Argentina & 1 & 1 & 0 & 2 & 55 & 0.63 \\
\hline Chile & 1 & 0 & 0 & 1 & 48 & 0.55 \\
\hline Uruguay & 1 & 1 & 1 & 3 & 46 & 0.53 \\
\hline Brazil & 4 & 0 & 0 & 4 & 44 & 0.50 \\
\hline Colombia & 3 & 0 & 1 & 4 & 35 & 0.40 \\
\hline Mexico & 0 & 2 & 1 & 3 & 34 & 0.39 \\
\hline Total & 34 & 32 & 3 & 69 & 8752 & 100 \\
\hline
\end{tabular}

${ }^{1}$ The total number of cases includes horses, donkeys and mules.

Figure 3 shows the flow diagram describing the cases included or excluded from the current analysis. The data cleaning process detected 193 empty data fields and $1310 \mathrm{in}-$ consistencies. After a discussion with the ambassador of each centre, 50 inconsistencies were redeemed, but were noted as unusual practices. Sixty two empty data fields and 1260 inconsistencies were corrected. For standing sedations, 131 durations were not found in the records of the centres and were coded as missing data. 
After data cleaning, 8752 cases ( 8656 horses, 92 donkeys and 4 mules) were confirmed. The median and range of cases received per day were 48 [1-146]. These preliminary results contain horse information only as the sample size for donkeys and mules is too small at this stage.

Of the 8656 horse cases, 6701 were general anaesthetics and 1955 were standing sedations. Of these, only 39 cases were procedures in the field, 31 total intravenous anaesthetics and 8 standing sedations. The demographic data for general anaesthetics and standing sedation are shown in Table 2.

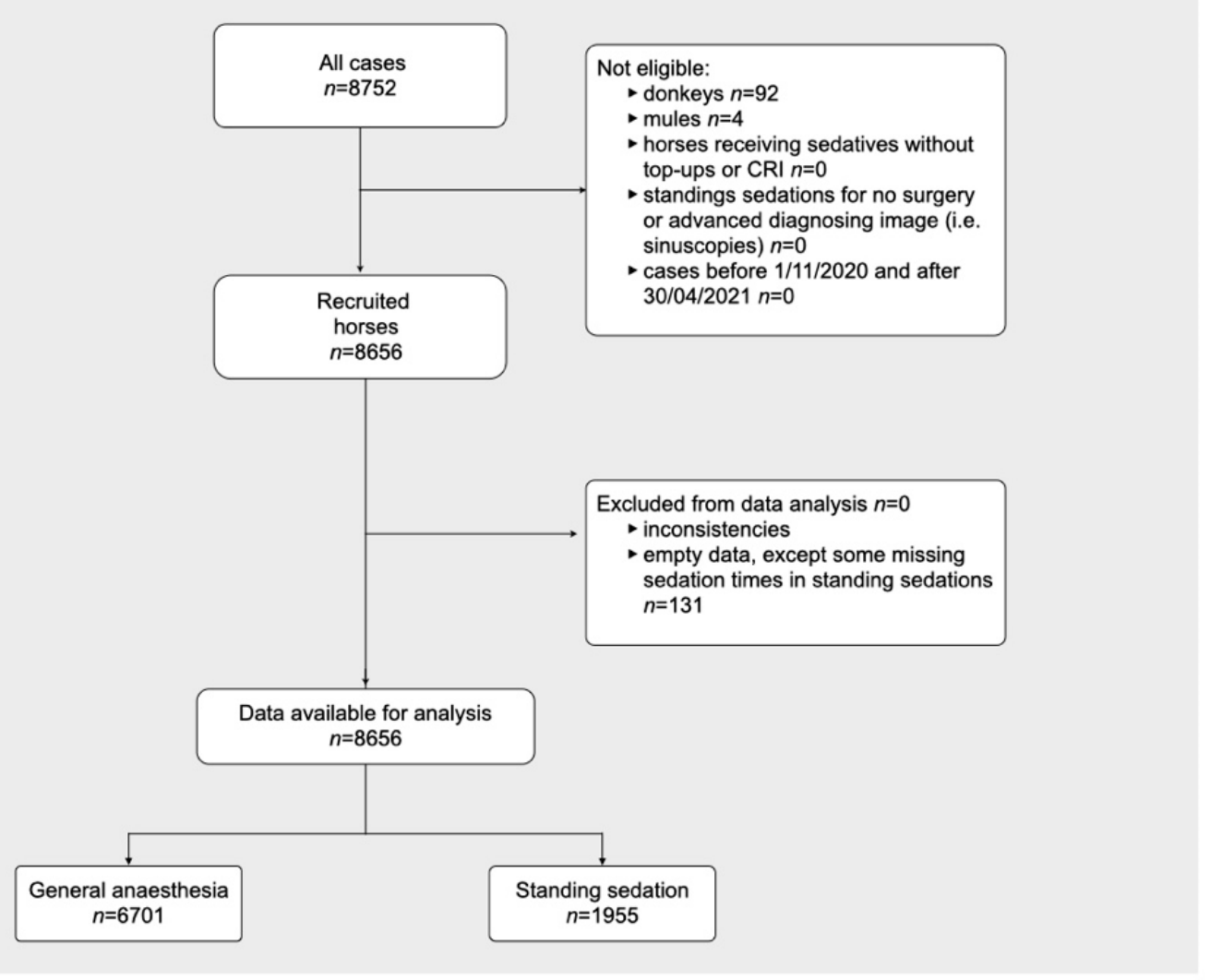

Figure 3. Flow diagram describing the cases included or excluded in the study. 
Table 2. Demographic data by general anaesthesia or standing sedation and overall, in 8656 horses.

\begin{tabular}{|c|c|c|c|c|}
\hline Variable & Categories & $\begin{array}{l}\text { General Anaesthesia } \\
\qquad(n=6701)\end{array}$ & $\begin{array}{l}\text { Standing Sedations } \\
\qquad(n=1955)\end{array}$ & $\begin{array}{c}\text { Overall } \\
(n=8656)\end{array}$ \\
\hline \multirow[t]{2}{*}{ Sex } & Female & 2699 & 817 & 3516 \\
\hline & Male & 4002 & 1138 & 5140 \\
\hline \multirow[t]{5}{*}{ Age } & Neonate ( $\leq 1$ month) & 229 & 30 & 259 \\
\hline & Foal (1-12 months) & 1407 & 27 & 1434 \\
\hline & Young (1-5 years) & 2314 & 328 & 2642 \\
\hline & Adult (5-14 years) & 2029 & 1131 & 3160 \\
\hline & Geriatric (>14 years) & 722 & 439 & 1161 \\
\hline \multirow[t]{3}{*}{ BCS } & Normal & 5766 & 1585 & 7351 \\
\hline & Thin & 415 & 129 & 544 \\
\hline & Fat & 520 & 241 & 761 \\
\hline \multirow[t]{5}{*}{ ASA } & I & 3007 & 1200 & 4207 \\
\hline & II & 2354 & 666 & 3020 \\
\hline & III & 546 & 79 & 625 \\
\hline & IV & 470 & 9 & 479 \\
\hline & $\mathrm{V}$ & 324 & 1 & 325 \\
\hline \multirow[t]{8}{*}{ Reason $^{1}$} & Abdominal & 218 & 61 & 279 \\
\hline & Colic & 917 & 6 & 923 \\
\hline & Diagnostic & 479 & 959 & 1438 \\
\hline & ENT & 377 & 138 & 515 \\
\hline & Fracture & 166 & 28 & 192 \\
\hline & Orthopaedics & 3346 & 138 & 3484 \\
\hline & Urogenital & 820 & 103 & 923 \\
\hline & Miscellaneous & 898 & 573 & 1471 \\
\hline \multirow{2}{*}{ Colic/noncolic } & Noncolic surgery & 5784 & 1949 & 7733 \\
\hline & Colic surgery & 917 & 6 & 923 \\
\hline \multirow[t]{4}{*}{ Type of anaesthesia } & Standing sedation & 0 & 1955 & 1955 \\
\hline & Inhalatory & 2282 & 0 & 2282 \\
\hline & PIVA & 3718 & 0 & 3718 \\
\hline & TIVA & 701 & 0 & 701 \\
\hline \multirow[t]{4}{*}{ Duration * } & $<1 \mathrm{~h}$ & 2317 & 594 & 2911 \\
\hline & $1-2 \mathrm{~h}$ & 2815 & 744 & 3559 \\
\hline & $2-3 \mathrm{~h}$ & 1123 & 333 & 1456 \\
\hline & $>3 \mathrm{~h}$ & 446 & 153 & 599 \\
\hline \multirow{2}{*}{ Use of locoregional techniques } & No & 5602 & 1114 & 6716 \\
\hline & Yes & 1099 & 841 & 1940 \\
\hline \multirow[t]{2}{*}{ Use of mechanical ventilation } & No & 1870 & 1955 & 3825 \\
\hline & Yes & 4831 & 0 & 4831 \\
\hline \multirow[t]{2}{*}{ Timetable } & Normal & 5866 & 1918 & 7784 \\
\hline & Out of hours & 835 & 37 & 872 \\
\hline \multirow[t]{3}{*}{ Scheduling } & Scheduled & 5120 & 1817 & 6937 \\
\hline & Non scheduled & 380 & 80 & 460 \\
\hline & Urgent & 1201 & 58 & 1259 \\
\hline
\end{tabular}

${ }^{1}$ Horses could be anaesthetized/sedated for more than one reason. ASA, American Society of Anaesthesiologists; BCS, body condition score; ENT, ear-nose-throat; PIVA, partial intravenous anaesthesia; TIVA, total intravenous anaesthesia. * 131 durations in standing sedations were coded as missing data. 


\subsection{Perioperative Equine Fatalitiess}

Sixty six of the 6701 horses that underwent general anaesthesia were classified as having died (1.0\%) (confidence interval 95\% (CI 95\%): 0.76-1.25\%). Of these, 35 (out of 5784 ) were classified as noncolic deaths (0.6\%) (CI 95\%: 0.42-0.84\%) and 31 (out of 917) as colic deaths (3.4\%) (CI 95\%: 2.3-4.8\%). Four of the 1955 horses undergoing standing sedation died (0.2\%); all were noncolic surgeries (0.2\%) (CI 95\%: 0.06-0.52\%).

Of the 6701 horses that underwent general anaesthesia, 329 were classified as PTS (4.9\%) (CI 95\%: 4.4-5.5\%): 76 of 5784 as noncolic PTS (1.3\%) (CI 95\%: $0.88-1.45 \%$ ) and 253 of 917 as colic PTS (27.6\%) (CI 95\%: 25.0-31.0\%). From the 1955 horses undergoing standing sedation, 13 were PTS (0.7\%) (CI 95\%: 0.35-1.13\%): 11 noncolic surgeries out of 1949 (0.6\%) (CI 95\%: 0.28-1.01\%) and 2 out of 6 colic surgeries (33.3\%) (CI 95\%: 4.3-77.7\%).

The outcomes at seven days post-anaesthesia or standing sedation are shown in Table 3. Details of the deaths classified as noncolics are presented in Table 4 . Table 5 indicates the time of death or PTS of all the cases whether general anaesthesia or standing sedation was used.

Table 3. Data from 6701 equine anaesthetics and 1955 equine standing sedations recorded: reason for anaesthesia/standing sedation and outcome at 7 days post-anaesthesia. Horses could be anesthetized or sedated for more than a reason.

\begin{tabular}{ccccccc}
\hline & Alive & Dead & \% Deaths & PTS & \% PTS & Total \\
\hline General anaesthesia & & & & & & \\
\hline Noncolic surgery & 5673 & 35 & $0.6 \%$ & 76 & $1.3 \%$ & 5784 \\
\hline Abdominal & 198 & 3 & $1.4 \%$ & 17 & $7.8 \%$ & 218 \\
Diagnostic & 440 & 3 & $0.6 \%$ & 36 & $7.5 \%$ & 479 \\
ENT & 373 & 4 & $1.1 \%$ & 0 & $0.0 \%$ & 377 \\
Fracture & 154 & 7 & $4.2 \%$ & 4 & $2.4 \%$ & 166 \\
Miscellaneous & 882 & 5 & $0.6 \%$ & 11 & $1.2 \%$ & 898 \\
Orthopaedics & 3306 & 13 & $0.4 \%$ & 27 & $0.8 \%$ & 3346 \\
Urogenital & 803 & 7 & $0.9 \%$ & 10 & $1.2 \%$ & 820 \\
\hline Colic surgery & 633 & 31 & $3.4 \%$ & 253 & $27.6 \%$ & 917 \\
\hline Overall & 6306 & 66 & $1.0 \%$ & 329 & $4.9 \%$ & 6701 \\
\hline Standing sedation & & & & & & \\
\hline Noncolic surgery & 1934 & 4 & $0.2 \%$ & 11 & $0.6 \%$ & 1949 \\
\hline Abdominal & 59 & 1 & $1.6 \%$ & 1 & $1.6 \%$ & 61 \\
Diagnostic & 950 & 0 & $0.0 \%$ & 9 & $0.9 \%$ & 959 \\
ENT & 137 & 0 & $0.0 \%$ & 1 & $0.7 \%$ & 138 \\
Fracture & 27 & 1 & $3.6 \%$ & 0 & $0.0 \%$ & 28 \\
Miscellaneous & 570 & 1 & $0.2 \%$ & 2 & $0.4 \%$ & 573 \\
Orthopaedics & 138 & 0 & $0.0 \%$ & 0 & $0.0 \%$ & 138 \\
Urogenital & 100 & 1 & $1.0 \%$ & 2 & $1.9 \%$ & 103 \\
\hline Colic surgery & 4 & 0 & $0.0 \%$ & 2 & $33.3 \%$ & 6 \\
\hline Overall & 1938 & 4 & $0.2 \%$ & 13 & $0.7 \%$ & 1955 \\
\hline ENT, ear & & & & & &
\end{tabular}

ENT, ear-nose-throat; PTS, "put to sleep" (euthanized) horses. 
Table 4. Noncolic deaths under general anaesthesia and standing sedations.

\begin{tabular}{|c|c|c|c|}
\hline Pre-Existing Condition and Brief Noncolic Death Description & $n$ & Phase & Protocol \\
\hline \multicolumn{4}{|l|}{ General anaesthesia } \\
\hline ASA I and II & 18 & & \\
\hline Fracture in recovery (2 ASA I, 3 ASA II) & 5 & Recovery & 2 PIVA, 2 INH, 1 TIVA \\
\hline Post-operative colic (5 ASA II) & 5 & Days $3,4,5,5$ and 5 & 3 PIVA, 2 INH \\
\hline Post-operative myelomalacia (2 ASA II) & 2 & Days 2 and 4 & 2 PIVA \\
\hline Cardiac arrest (ASA I) & 1 & Maintenance & INH \\
\hline Upper airway obstruction in recovery (ASA II) & 1 & Recovery & PIVA \\
\hline Fracture C2 (ASA II) & 1 & Recovery & PIVA \\
\hline Sudden collapse after standing (ASA I) & 1 & Recovery & TIVA \\
\hline Presumed spinal cord malacia (ASA II) & 1 & Recovery & PIVA \\
\hline Small colon prolapse (ASA II) & 1 & Day 1 & PIVA \\
\hline ASA III, IV and V & 17 & & \\
\hline Re-fractures in recovery (3 ASA IV) & 3 & Recovery & 3 PIVA \\
\hline Recumbent on arrival. Unable to stand after anaesthesia (2 ASA III) & 2 & Recovery & 1 PIVA, 1 TIVA \\
\hline Intraoperative bleeding without response to treatment (2 ASA III) & 2 & Maintenance & 2 PIVA \\
\hline Catastrophic recovery (<1 year with sling) (ASA III) & 1 & Recovery & PIVA \\
\hline Post-operative colic (1 ASA III) & 1 & Day 3 & INH \\
\hline Re-fracture olecranon in the stable (ASA III) & 1 & Day 3 & PIVA \\
\hline Unknown, could not stand, no fracture diagnosed in X-rays (ASA III) & 1 & Recovery & INH \\
\hline $\begin{array}{l}\text { Severe chronic sinusitis. Sudden death. Necropsy: communication } \\
\text { between sinus fistula and cranial cavity (ASA IV) }\end{array}$ & 1 & Recovery & TIVA \\
\hline Foal with sepsis, diarrhoea (ASA IV) & 1 & Day 5 & TIVA \\
\hline Bladder rupture on caesarean section (ASA V) & 1 & Maintenance & PIVA \\
\hline Fracture rib repair, hemothorax and hemopericardium (ASA V) & 1 & Maintenance & $\mathrm{INH}$ \\
\hline Fracture/abductor tear after dystocia (ASA V) & 1 & Day 2 & PIVA \\
\hline Intraoperative bleeding dystocia (ASA V) & 1 & Day 1 & INH \\
\hline Standing sedation & 4 & & \\
\hline Post-operative colitis (ASA I, ASA II) & 2 & Days 1 and 6 & CRI \\
\hline Post-operative colic (ASA III) & 1 & Day 2 & CRI \\
\hline Re-fracture in stable (ASA III) & 1 & Day 1 & CRI \\
\hline
\end{tabular}

ASA, American Society of Anaesthesiologists; CRI, continuous rate infusion; INH, inhalational anaesthesia only; PIVA, partial intravenous anaesthesia; TIVA, total intravenous anaesthesia.

Table 5. Time of death of horses under general anaesthesia and standing sedation.

\begin{tabular}{ccccccccccccc}
\hline \multirow{2}{*}{ Colic } & PREM & IND & MAIN & REC & 1D & 2D & 3D & 4D & 5D & 6D & 7D \\
& Deaths & 1 & 3 & 7 & 10 & 6 & 1 & 1 & 1 & 0 & 0 & 1 \\
& PTS & 0 & 0 & 186 & 12 & 14 & 15 & 9 & 5 & 9 & 0 & 5 \\
\hline \multirow{2}{*}{ Noncolic } & Deaths & 0 & 0 & 5 & 18 & 4 & 3 & 3 & 1 & 4 & 1 & 0 \\
& PTS & 0 & 0 & 38 & 3 & 15 & 12 & 8 & 4 & 3 & 1 & 3 \\
\hline \multirow{2}{*}{ Total } & Deaths & 1 & 3 & 12 & 28 & 10 & 4 & 4 & 2 & 4 & 1 & 1 \\
& PTS & 0 & 0 & 224 & 15 & 29 & 27 & 17 & 9 & 12 & 1 & 8
\end{tabular}

IND, induction; MAIN, maintenance; PREM, premedication; REC, recovery period; PTS, "put to sleep" (euthanized) horses. 1D-7D: days 1st to 7th.

\subsection{General Anaesthesia}

The centres sent a median of $100 \%$ of cases (100 [95-100]\%). Of the 69 centres, 63 confirmed that $100 \%$ of the cases were sent, three sent more than $98 \%$ and three sent more than $95 \%$.

Table 6 gives the details of the individual drugs used at each phase of general anaesthesia. The protocols for the general anaesthetics are described in Tables 7-11. Information about the method of induction and recovery from general anaesthesia is shown in Tables 12 and 13, respectively. Figure 4 describes the monitoring of the horses under general anaesthesia. 
Table 6. List of the individual drugs used at each phase of general anaesthesia in a total of 6701 horses.

\begin{tabular}{|c|c|c|c|c|c|}
\hline Drugs & PREM & IND & $\begin{array}{l}\text { MAIN-(Bolus } \\
\text { If Not Inhalant) }\end{array}$ & MAIN-CRI & POST \\
\hline Acepromazine & 2776 & 0 & 42 & 0 & 162 \\
\hline Xylazine & 3231 & 0 & 327 & 724 & 2303 \\
\hline Detomidine & 1569 & 0 & 44 & 274 & 340 \\
\hline Romifidine & 1874 & 0 & 89 & 1132 & 1699 \\
\hline Medetomidine & 236 & 0 & 81 & 471 & 287 \\
\hline Dexmedetomidine & 5 & 0 & 65 & 296 & 52 \\
\hline Midazolam & 87 & 2795 & 40 & 125 & 2 \\
\hline Diazepam & 112 & 3293 & 17 & 0 & 2 \\
\hline Morphine & 1270 & 0 & 239 & 7 & 222 \\
\hline Methadone & 498 & 0 & 66 & 2 & 26 \\
\hline Pethidine & 0 & 0 & 0 & 0 & 0 \\
\hline Fentanyl & 6 & 0 & 4 & 3 & 0 \\
\hline Buprenorphine & 2 & 0 & 1 & 0 & 5 \\
\hline Butorphanol & 3320 & 0 & 261 & 238 & 94 \\
\hline Phenylbutazone & 2647 & 0 & 64 & 0 & 657 \\
\hline Flunixin & 2223 & 0 & 73 & 0 & 673 \\
\hline Meloxicam & 413 & 0 & 5 & 0 & 143 \\
\hline Ketoprofen & 93 & 0 & 1 & 0 & 6 \\
\hline Propofol & 0 & 316 & 40 & 8 & 21 \\
\hline Alfaxalone & 0 & 0 & 1 & 0 & 0 \\
\hline Ketamine & 0 & 6633 & 1158 & 1090 & 37 \\
\hline Thiopental & 0 & 95 & 234 & 0 & 23 \\
\hline TLT-ZLZ & 0 & 41 & 1 & 0 & 0 \\
\hline GGE & 0 & 84 & 44 & 442 & 0 \\
\hline Halothane & 0 & 0 & 0 & 0 & 0 \\
\hline Isoflurane & 0 & 1 & 5250 & 0 & 0 \\
\hline Sevoflurane & 0 & 1 & 547 & 0 & 0 \\
\hline Desflurane & 0 & 1 & 203 & 0 & 0 \\
\hline Lidocaine & 17 & 0 & 0 & 1255 & 86 \\
\hline Dobutamine & 0 & 0 & 0 & 4281 & 20 \\
\hline Phenylephrine & 0 & 0 & 12 & 277 & 960 \\
\hline
\end{tabular}

CRI, continuous rate infusion; GGE, guaiacol glyceryl ether; IND, induction; MAIN, maintenance; POST, immediate postoperative period; PREM, premedication; TLT-ZLZ, tiletamine-zolazepam.

Table 7. Drugs and different drug combinations used for premedication before general anaesthesia in 6701 horses.

\begin{tabular}{|c|c|c|}
\hline Drugs and Combinations & $n$ & $\%$ \\
\hline $\mathrm{Alpha}_{2}+$ Partial/Agonists-Antagonists Opioids & 2214 & $33.0 \%$ \\
\hline ACP + Alpha $2+$ Partial/Agonists-Antagonists Opioids & 1026 & $15.3 \%$ \\
\hline $\mathrm{ACP}+\mathrm{Alpha}_{2}+$ Pure Opioids & 1017 & $15.2 \%$ \\
\hline Alpha 2 alone & 872 & $13.0 \%$ \\
\hline $\mathrm{ACP}+\mathrm{Alpha}_{2}$ & 721 & $10.8 \%$ \\
\hline $\mathrm{Alpha}_{2}+$ Pure Opioids & 704 & $10.5 \%$ \\
\hline Partial/Agonist-Antagonist Opioid alone & 62 & $0.9 \%$ \\
\hline Pure Opioid alone & 31 & $0.5 \%$ \\
\hline Benzodiazepine alone & 27 & $0.4 \%$ \\
\hline $\mathrm{ACP}+\mathrm{Alpha}_{2}+$ Pure Opioids + Partial/Agonists-Antagonists Opioids & 11 & $0.2 \%$ \\
\hline Alpha $_{2}+$ Pure Opioids + Partial/Agonists-Antagonists Opioids & 8 & $0.1 \%$ \\
\hline ACP + Partial/Agonists-Antagonists Opioids & 1 & $0.0 \%$ \\
\hline None & 7 & $0.1 \%$ \\
\hline
\end{tabular}

ACP, acepromazine; Alpha 2 , Alpha 2 -agonists. Benzodiazepines: $199-27$ = 172 were used in different combinations but not alone. 
Table 8. Drugs used for induction of general anaesthesia in 6701 horses.

\begin{tabular}{ccc}
\hline Induction Drugs & $\boldsymbol{n}$ & $\%$ \\
\hline Ketamine + Benzodiazepine & 5919 & $88.3 \%$ \\
Ketamine + Propofol & 295 & $4.4 \%$ \\
Ketamine alone & 247 & $3.7 \%$ \\
Ketamine + Thiopental & 91 & $1.4 \%$ \\
Ketamine + GGE & 80 & $1.2 \%$ \\
Tiletamine + Zolazepam & 41 & $0.6 \%$ \\
Propofol & 21 & $0.3 \%$ \\
Thiopental + GGE & 3 & $0.1 \%$ \\
Ketamine + Inhalatory & 1 & $0.0 \%$ \\
Inhalatory + GGE & 1 & $0.0 \%$ \\
Thiopental alone & 1 & $0.0 \%$ \\
Inhalatory & 1 & $0.0 \%$
\end{tabular}

GGE, guaiacol glyceryl ether. Benzodiazepines: 6088 - 5919 = 169 were used in different combinations but benzodiazepines + ketamine.

Table 9. Drugs used for maintenance of general anaesthesia in 6701 horses.

\begin{tabular}{ccc}
\hline Maintenance Drugs & $\boldsymbol{n}$ & $\mathbf{\%}$ \\
\hline Isoflurane & 5250 & $78.3 \%$ \\
Sevoflurane & 547 & $8.2 \%$ \\
Triple drip alone & 277 & $4.1 \%$ \\
Desflurane & 203 & $3.0 \%$ \\
Ketamine alone & 87 & $1.3 \%$ \\
Thiopental alone & 4 & $0.1 \%$ \\
Ketamine + Thiopental & 3 & $0.0 \%$ \\
None & 330 & $5.0 \%$ \\
\hline
\end{tabular}

Table 10. Drugs used for continuous rate infusion during general anaesthesia with partial intravenous anaesthesia (PIVA) in 3718 horses.

\begin{tabular}{|c|c|c|}
\hline CRI Drugs & $n$ & $\%$ \\
\hline Alpha 2 alone & 1780 & $47.9 \%$ \\
\hline Lidocaine alone & 1041 & $28.0 \%$ \\
\hline $\mathrm{Alpha}_{2}+$ Ketamine & 384 & $10.3 \%$ \\
\hline Alpha $_{2}+$ Ketamine + Butorphanol & 147 & $4.0 \%$ \\
\hline Alpha $_{2}+$ Lidocaine & 78 & $2.1 \%$ \\
\hline Ketamine alone & 70 & $1.9 \%$ \\
\hline Alpha $_{2}+$ Butorphanol & 68 & $1.8 \%$ \\
\hline Lidocaine + Ketamine & 65 & $1.7 \%$ \\
\hline Alpha $_{2}+$ Lidocaine + Ketamine & 47 & $1.3 \%$ \\
\hline Alpha $_{2}+$ Lidocaine + Butorphanol & 18 & $0.5 \%$ \\
\hline Other combinations & 20 & $0.5 \%$ \\
\hline
\end{tabular}

Alpha 2, Alpha $_{2}$ agonists; CRI, continuous rate infusion. 
Table 11. Parenteral drugs administered for/during the immediate recovery period after general anaesthesia in 6701 horses.

\begin{tabular}{ccc}
\hline Drugs and Combinations & $\boldsymbol{n}$ & $\%$ \\
\hline Alpha 2 alone & 4220 & $63.0 \%$ \\
Alpha + Pure Opioids & 197 & $2.9 \%$ \\
ACP + Alpha 2 & 91 & $1.4 \%$ \\
ACP alone & 65 & $1.0 \%$ \\
Pure Opioid alone & 38 & $0.6 \%$ \\
Alpha 2 + Partial/Agonists-Antagonists Opioids & 37 & $0.6 \%$ \\
ACP + Alpha 2 + Partial/Agonists-Antagonists Opioids & 18 & $0.3 \%$ \\
Partial/Agonist-Antagonist Opioid alone & 11 & $0.2 \%$ \\
ACP + Alpha 2 + Pure Opioids & 10 & $0.1 \%$ \\
ACP + Partial/Agonists-Antagonists Opioids & 3 & $0.0 \%$ \\
Benzodiazepine alone & 2 & $0.0 \%$ \\
ACP + Alpha + Pure Opioids + Partial/Agonists-Antagonists Opioids & 1 & $0.0 \%$ \\
ACP + Pure Opioids & 1 & $0.0 \%$ \\
Pure Opioids + Partial/Agonists-Antagonists Opioids & 1 & $0.0 \%$ \\
None & 2006 & $29.9 \%$
\end{tabular}

ACP, acepromazine; Alpha 2 , Alpha 2 agonists. Alpha 2 -agonists: 79 cases received more than one alpha ${ }_{2}$-agonist in the recovery.

Table 12. Methods of induction of general anaesthesia for 6701 horses under inhalational anaesthesia only (INH), partial intravenous anaesthesia (PIVA), total intravenous anaesthesia (TIVA) and overall.

\begin{tabular}{ccccc}
\hline Induction & $\begin{array}{c}\text { INH } \\
(\boldsymbol{n}=\mathbf{2 2 8 2})\end{array}$ & $\begin{array}{c}\text { PIVA } \\
(\boldsymbol{n}=\mathbf{3 7 1 8})\end{array}$ & $\begin{array}{c}\text { TIVA } \\
(\boldsymbol{n}=\mathbf{7 0 1})\end{array}$ & $\begin{array}{c}\text { Overall } \\
(\boldsymbol{n}=\mathbf{6 7 0 1 )})\end{array}$ \\
\hline Personnel assisted & $1498(65.6 \%)$ & $2010(54.1 \%)$ & $570(81.3 \%)$ & $4078(60.8 \%)$ \\
Gate & $353(15.5 \%)$ & $1207(32.5 \%)$ & $86(12.3 \%)$ & $1646(24.6 \%)$ \\
Free & $407(17.8 \%)$ & $479(12.9 \%)$ & $44(6.3 \%)$ & $930(13.9 \%)$ \\
Table & $18(0.8 \%)$ & $9(0.2 \%)$ & $0(0 \%)$ & $27(0.4 \%)$ \\
Sling & $6(0.3 \%)$ & $13(0.3 \%)$ & $1(0.1 \%)$ & $20(0.3 \%)$ \\
\hline
\end{tabular}

Table 13. Methods of recovery from general anaesthesia for 6461 horses after inhalational anaesthesia only (INH), partial intravenous anaesthesia (PIVA), total intravenous anaesthesia (TIVA) and overall.

\begin{tabular}{ccccc}
\hline & $\begin{array}{c}\text { INH } \\
(\boldsymbol{n}=\mathbf{2 2 2 5})\end{array}$ & $\begin{array}{c}\text { PIVA } \\
(\boldsymbol{n}=\mathbf{3 5 4 3 )}\end{array}$ & $\begin{array}{c}\text { TIVA } \\
(\boldsymbol{n}=\mathbf{6 9 3})\end{array}$ & $\begin{array}{c}\text { Overall * } \\
(\boldsymbol{n}=\mathbf{6 4 6 1 )}\end{array}$ \\
\hline Free & $1049(47.2 \%)$ & $1770(50.0 \%)$ & $462(66.7 \%)$ & $3281(50.8 \%)$ \\
Ropes & $942(42.3 \%)$ & $1598(45.1 \%)$ & $116(16.7 \%)$ & $2656(41.1 \%)$ \\
Manual & $234(10.5 \%)$ & $175(4.9 \%)$ & $115(16.6 \%)$ & $524(8.1 \%)$ \\
\hline
\end{tabular}

* $6701-6461=240$ horses that were put to sleep (PTS) or died before recovery (i.e., premedication, induction or maintenance). 


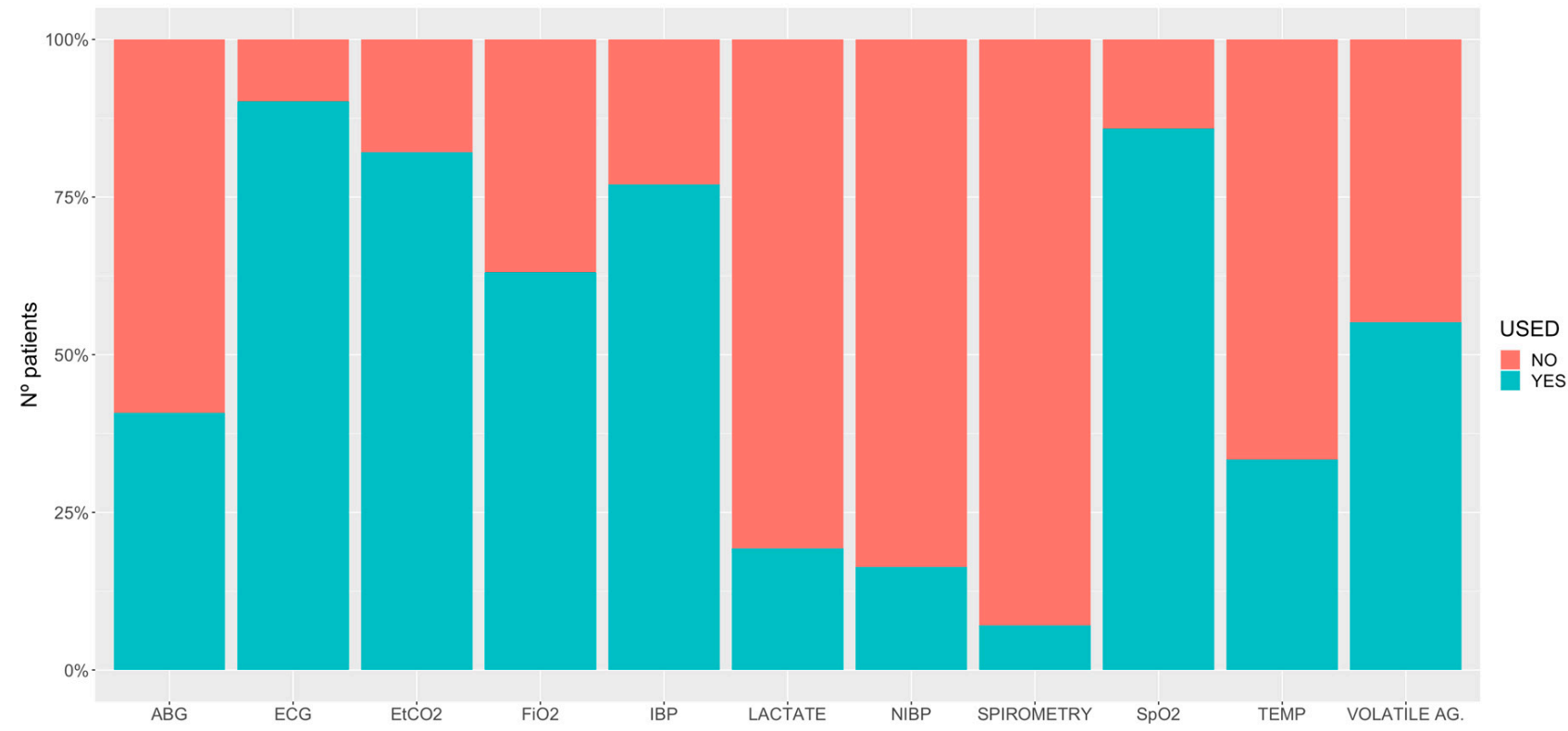

Figure 4. Percentage of monitoring used in 6701 horses undergoing general anaesthesia. ABG, arterial blood gases; ECG, electrocardiogram; $\mathrm{E}_{\mathrm{t}} \mathrm{CO}_{2}$, end-tidal carbon dioxide; $\mathrm{FiO}_{2}$, inspiratory fraction of oxygen; IBP, invasive blood pressure; NIBP, non-invasive blood pressure; $\mathrm{SpO}_{2}$, partial saturation of haemoglobin with oxygen by pulse-oximetry; TEMP, temperature; VOLATILE AG., volatile agents measured for inspired fraction and end-tidal concentration.

\subsection{Standing Sedation}

From the 69 collaborating centres, 57 sent standing sedation cases that fulfilled our inclusion criteria (median 99 [10-100]\%). Forty-three centres sent more than $90 \%$ of their cases, four sent between 80 and 90\%, five sent between 50 and $80 \%$ and five sent between 1 and $49 \%$.

The drugs and protocols for standing sedations are shown in Tables 14 and 15, respectively. Table 16 reports the different CRIs used to maintain standing sedation. Figure 5 describes the monitoring of the horses under sedation.

Table 14. Drugs used for standing sedation in 1955 horses.

\begin{tabular}{ccccc}
\hline Drugs & PREM & MAIN-(Bolus) & MAIN-CRI & POST \\
\hline Acepromazine & 918 & 15 & 0 & 1 \\
Xylazine & 61 & 17 & 29 & 1 \\
Detomidine & 1574 & 1178 & 525 & 1 \\
Romifidine & 405 & 302 & 60 & 0 \\
Medetomidine & 11 & 8 & 10 & 0 \\
Dexmedetomidine & 4 & 7 & 4 & 0 \\
Midazolam & 15 & 61 & 34 & 0 \\
Diazepam & 3 & 10 & 1 & 0 \\
Morphine & 532 & 54 & 76 & 2 \\
Methadone & 223 & 20 & 4 & 0 \\
Pethidine & 2 & 0 & 0 & 0 \\
Fentanyl & 2 & 0 & 0 & 0 \\
Buprenorphine & 3 & 0 & 94 & 0 \\
Butorphanol & 922 & 437 & 0 & 115 \\
Phenylbutazone & 253 & 5 & 0 & 137 \\
Flunixin & 513 & 3 & 0 & 14 \\
Meloxicam & 39 & 2 & 24 & 0 \\
Ketamine & 0 & 8 & 3 & 14 \\
Phenylephrine & 0 & 1 & 0 . & \\
\hline CRI, continuous rate infusion; MAIN, maintenance; POST, postoperative period; PREM, premedication.
\end{tabular}


Table 15. Drugs and different drug combinations used for premedication before standing sedations in 1955 horses.

\begin{tabular}{|c|c|c|}
\hline Drugs and Combinations & $n$ & $\%$ \\
\hline $\mathrm{Alpha}_{2}+$ Partial/Agonists-Antagonists Opioids & 588 & $30.1 \%$ \\
\hline $\mathrm{ACP}+\mathrm{Alpha}_{2}+$ Pure Opioids & 393 & $20.1 \%$ \\
\hline Alpha $_{2}+$ Pure Opioids & 338 & $17.3 \%$ \\
\hline $\mathrm{ACP}+\mathrm{Alpha}_{2}+$ Partial/Agonists-Antagonists Opioids & 312 & $16.0 \%$ \\
\hline $\mathrm{ACP}+\mathrm{Alpha}_{2}$ & 203 & $10.4 \%$ \\
\hline $\mathrm{Alpha}_{2}$ alone & 93 & $4.8 \%$ \\
\hline Alpha $_{2}+$ Pure Opioids + Partial/Agonists-Antagonists Opioids & 14 & $0.7 \%$ \\
\hline ACP + Alpha $2+$ Pure Opioids + Partial/ Agonists-Antagonists Opioids & 10 & $0.5 \%$ \\
\hline Pure Opioid alone & 3 & $0.1 \%$ \\
\hline Partial/Agonist-Antagonist Opioid alone & 1 & $0.0 \%$ \\
\hline
\end{tabular}

ACP, acepromazine; $\mathrm{Alpha}_{2}, \mathrm{Alpha}_{2}$ agonists.

Table 16. Drugs and different drug combinations used for continuous rate infusion in standing sedations in 1955 horses.

\begin{tabular}{ccc}
\hline CRI Drugs & $n$ & $\%$ \\
\hline Alpha alone & 448 & $22.9 \%$ \\
Alpha 2 Butorphanol & 88 & $4.5 \%$ \\
Alpha 2 - Morphine & 66 & $3.4 \%$ \\
Alpha 2 Ketamine & 10 & $0.5 \%$ \\
Alpha $_{2}+$ Ketamine + Morphine & 8 & $0.4 \%$ \\
Alpha + Ketamine + Butorphanol & 4 & $0.2 \%$ \\
Alpha 2 + Methadone & 3 & $0.2 \%$ \\
Other combinations & 6 & $0.3 \%$ \\
No CRI (only top-ups) & 1322 & $67.6 \%$ \\
\hline
\end{tabular}

$\mathrm{Alpha}_{2}, \mathrm{Alpha}_{2}$ agonists; CRI, continuous rate infusion.
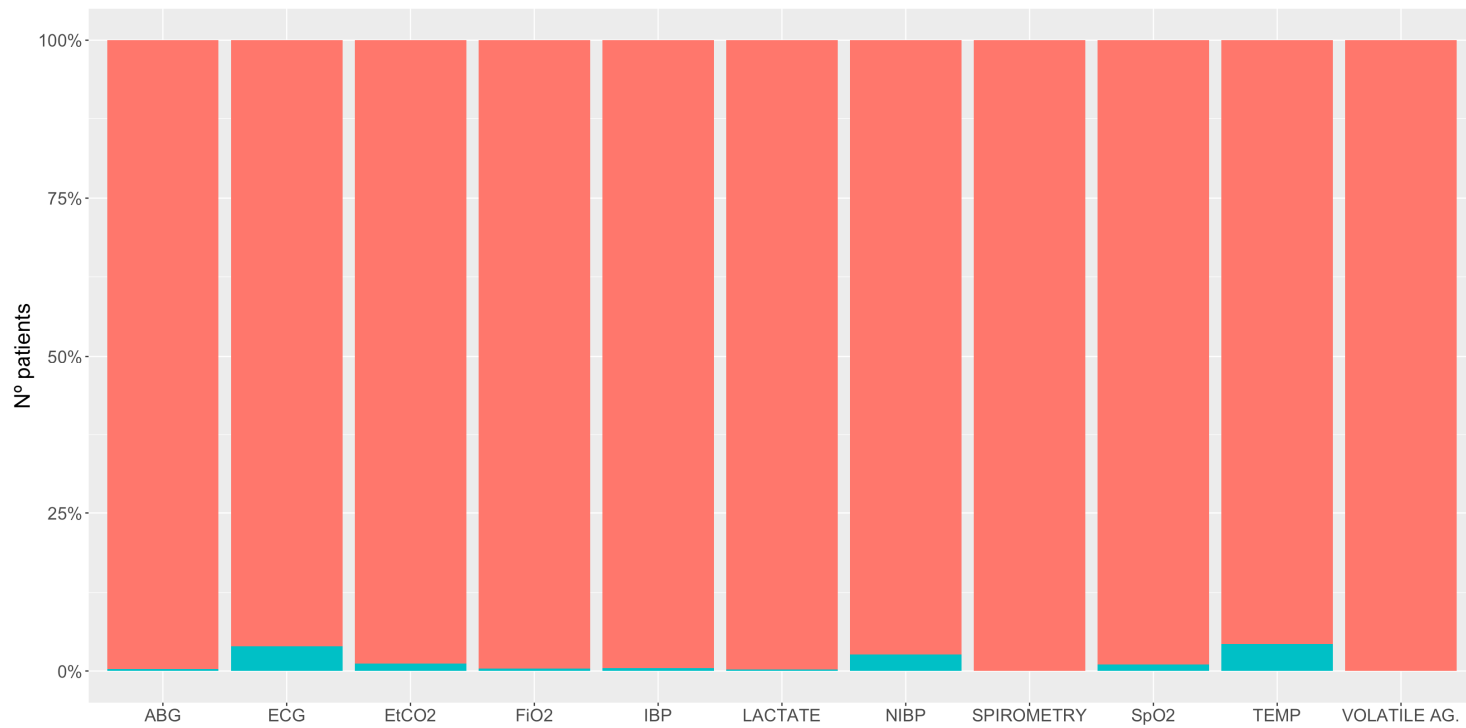

USED

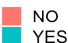

Figure 5. Percentage of monitoring used in 1955 horses undergoing standing sedation. ABG, arterial blood gases; ECG, electrocardiogram; $\mathrm{E}_{\mathrm{t}} \mathrm{CO}_{2}$, end-tidal carbon dioxide; $\mathrm{FiO}_{2}$, inspiratory fraction of oxygen; IBP, invasive blood pressure; NIBP, non-invasive blood pressure; $\mathrm{SpO}_{2}$, partial saturation of haemoglobin with oxygen by pulse-oximetry; TEMP, temperature; VOLATILE AG., volatile agents measured for inspired fraction and end-tidal concentration.

\subsection{Results of Survey for Feedback in Final Meeting}

The ambassadors of the collaborating centres gave a final mark for the project and the communication process of median 10 [7-10] (0-worse to 10-excellent). 


\section{Discussion}

The results presented here demonstrate the utility of the electronic questionnaire and the internet-based method for data collection, with interactive data handling and cleaning for the performance of the CEPEF4 study. The data collected from the 1 November 2020 until the 30 April 2021 show that overall, 1\% of horses undergoing general anaesthesia still die within the seven-day outcome period. Even with this preliminary evaluation of a small number of horses, it appears that a fatal outcome in both noncolics $(0.6 \%)$ and colics (3.4\%) was less frequent than that reported by Johnston et al. almost 20 years ago [18]. As anticipated, and despite the higher risk of bias collecting standing sedation data, the risk of death with standing sedation $(0.2 \%)$ appears lower than with general anaesthesia. However, even with a lower risk, some noncolic horses died unexpectedly when undergoing standing sedation within the seven-day outcome period. The data also demonstrate certain changes in the routine anaesthetic practice and protocols that have developed since CEPEF2.

In light of our findings, and focussing on our main objectives, the discussion is structured in the following four parts: (1) the proposed method for data collection with interactive data handling and cleaning, (2) the outcomes of horses that underwent general anaesthesia and standing sedation, (3) the preliminary data from horses undergoing general anaesthesia and (4) the preliminary results of standing sedation in horses.

(1) The proposed method for data collection with interactive data handling and cleaning

Our first hypothesis was confirmed as the proposed internet-based method proved to be a reliable, easy, quick and cheap means of collecting data, with minimal geographical limitations. This preliminary phase of CEPEF4 was initiated and executed during the COVID19 pandemic but our methodology allowed us to communicate with the collaborating centres and to collect and handle data arising from many locations worldwide. The feedback from the ambassadors of each centre indicated that the communication process (recruitment, followup and final meetings) allowed fluent communication that undoubtedly contributed to the collection of good quality data [2]. Once collected, our R scripts detected many inconsistences that were reduced almost to zero prior to final submission. The three steps followed for this data cleaning proved effective: first, data screening to detect missing or excess data, outliers, inconsistencies or strange patterns. Second, diagnosis to detect missing data, true extremes or true errors. Finally, data editing or treatment to correct, delete or leave unchanged the detected inconsistencies [5] as agreed with each centre's ambassadors. Even with this large dataset, we achieved a reliable method with a clear strategy for communication and data collection/cleaning. This approach will help to minimize inconsistencies that might lead to data misinterpretation for the current CEPEF4 study.

For the first six months, we compiled 8656 horse cases: 6701 general anaesthetics and 1955 standing sedations. CEPEF1 collected 6255 general anaesthetics in a period of two years (February 1991-March 1993), using paper copies and communication by post and telephone [16]. This reflects the importance of new technologies that allow interactive communication, data collection and analysis. Furthermore, the rapid availability of a community of researchers and equine clinicians familiar with the CEPEF studies made this work possible in such a short period of time. The impact of the CEPEF2 study, cited by 448 other publications (according to Google Scholar on the 29 August 2021), reflects the importance of an update on these data [20].

With the first hypothesis confirmed, our final goal is to use this methodology to collect approximately 45,000 cases of general anaesthetics for CEPEF4, to increase the statistical power and to compare the results with those of 20 years ago [18]. In order to ensure a robust comparison, we followed the same approach as in CEPEF2. Both M.J. and P.M.T., authors of the previous series, carefully supervised the study design and the subsequent implementation of data collection and cleaning. On the basis of the cumulative cases collected per week to date, the proposed cases numbers should be reached in approximately two years. 
(2) Outcome of horses under general anaesthesia and standing sedation

As reported in CEPEF2 [18], the overall equine anaesthetic mortality rate is still higher than in other veterinary species such as dogs and cats [30]. However, these preliminary data from a small population do suggest that the current rate is lower than 20 years ago.

As stated in 2016 by Dugdale and Taylor [19], "we still lose horses after anaesthesia to a range of catastrophes that would not occur if the horse were not anaesthetized". Our preliminary results confirm this statement. We recorded 39 horses that were classified as noncolic deaths: 34 general anaesthetics, four standing sedations and one that started as standing sedation and later changed to general anaesthesia.

Focusing on the noncolics undergoing general anaesthesia, with the exception of 17 cases classified as moderate- or high-risk patients (ASA III, IV or V), the remaining 18 were healthy (four ASA I, 14 ASA II). This indicates that noncolic horses undergoing general anaesthesia still die unexpectedly; $0.6 \%$ at this stage, versus the $0.9 \%$ of CEPEF2. Further recruitment of cases to achieve our final CEPEF4 goal should help to clarify why this occurs and hopefully suggest how these numbers could be reduced.

With regard to colics undergoing general anaesthesia, our preliminary data recorded $3.4 \%$ that died, compared to the $7.8 \%$ (457 colic deaths out of 5846) in CEPEF2. This relative reduction may indicate an improvement in the peri-operative management of horses with colic. However, the number of colic PTSs remain extremely high, $27.6 \%$ in this data versus the $25.2 \%$ of CEPEF2.

It is generally assumed that standing procedures are safer than general anaesthesia in horses. However, this is the first study to report mortality rates associated with standing sedation in a multicentre, prospective, cohort study. The four standing sedations (one ASA I, one ASA II and two ASA III) classified as noncolic deaths $(0.2 \%)$ provide evidence that standing sedation procedures still carry a risk, albeit less than general anaesthesia.

(3) Preliminary results for general anaesthetics in horses

One third of the horses were premedicated with a combination of an alpha 2 -agonist and a partial/agonist-antagonist opioid, mainly butorphanol. When acepromazine was also given, the percentage increased to $48 \%$. Alpha 2 -agonists with a pure opioid, with or without acepromazine, was used in about $26 \%$ of the cases. Only $13 \%$ of the cases received

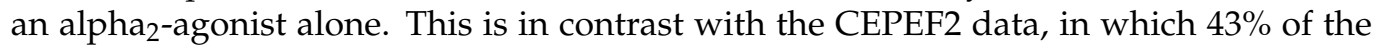
horses received an alpha 2 -agonist alone, 29\% combined acepromazine with an alpha $2^{-}$ agonist and no more than $8 \%$ included opioids such us methadone or butorphanol [18]. Our data show the tendency for premedication with drug combinations rather than a single agent. Drug combinations calm the horse, enhance sedation and provide analgesia by employing low doses of each drug, which reduces their potential side effects [31-33]. Compared with around 16\% in CEPEF2 [18], premedication with acepromazine alone has not been reported in the current study.

Ketamine was the most commonly used intravenous agent for the induction of general anaesthesia, usually combined with a benzodiazepine as a central muscle relaxant $(88 \%)$. Diazepam was frequently used, and increasingly midazolam, which was not reported at all in CEPEF2. Midazolam has recently gained Market Authorisation for use in horses in Europe, which would support its increased use. Thiopental and guaiphenesin, an intravenous anaesthetic and a central muscle relaxant, respectively, were rarely used. This combination was historically the evolution from chloral hydrate and pentobarbital [34]; however, the often prolonged and violent recovery from barbiturate anaesthesia provoked the transition towards ketamine [35]. A few techniques were popular in some individual clinics, although the overall numbers were not high. For example, the induction and brief maintenance of general anaesthesia in foals with propofol, sometimes combined with ketamine [36].

Isoflurane was the most common inhalation agent used for the maintenance of general anaesthesia $(88 \%)$, followed by sevoflurane $(9 \%)$ and desflurane $(3 \%)$. None of the anaesthetics reported in these preliminary results used halothane in contrast with the previous CEPEF studies [16,18]. In 2004, halothane was considered an acceptable anaesthetic for 
the maintenance of anaesthesia in horses [37]; however, it is no longer manufactured and is now rarely used. Our data also showed the current tendency to use an inhalant agent in combination with an intravenous CRI, the so-called partial intravenous anaesthesia (PIVA) [38-40]. Out of all the 6000 inhalant-based general anaesthetics, 3718 were PIVA $(62 \%)$ versus $2282(38 \%)$ that were pure inhalation anaesthesia. Since the publication of CEPEF2 nearly 20 years ago, there have been numerous reports of PIVA techniques using alpha 2 -adrenergic agonists [41,42], lidocaine [43], ketamine [44] and opioids [45,46].

Only 701 of the total 6701 were general anaesthetics where total intravenous anaesthesia (TIVA) was used, 31 under field conditions. Very short procedures were carried out in the anaesthetic recovery box or in the field using repeated boluses of a range of drugs and combinations. Ketamine, thiopental, alpha ${ }_{2}$-agonists and benzodiazepines were all used. Less than 300 of the 701 TIVA cases were maintained with various combinations of the so-called "triple drip", usually combining ketamine with an alpha ${ }_{2}$-agonist and guaiphenesin [40]. The maintenance of anaesthesia for short procedures was also carried out with ketamine alone or occasionally with thiopental.

Our data show that it is now common practice $(63 \%)$ to administer a small dose of an alpha 2 -agonist after the end of general anaesthesia before the recovery phase, either when the patient is still on the surgical table and before transport to the recovery box, or once in the recovery box. Santos et al. (2003) [47] first demonstrated its benefits, later confirmed by others [48,49].

This study collected relevant information about the monitoring undertaken during general anaesthesia. Although it has been recently suggested that improvements in monitoring have reduced the risk of anaesthetic mortality [19], no data from a prospective, multicentre study support this statement. Our data indicated that around $90 \%$ of the horses undergoing general anaesthesia (INH, PIVA or TIVA) had an electrocardiogram and a pulse oximeter. Arterial blood pressure (invasively) and the end-tidal carbon dioxide $\left(\mathrm{EtCO}_{2}\right)$ concentration were measured in more than $75 \%$. Inspired oxygen and an end tidal volatile agent were measured in fewer cases, but still more than $50 \%$. Arterial blood gas analyses $(70 \%)$ and lactate $(43 \%)$ were performed most often in horses with colic. In general, body temperature was not frequently monitored $(33 \%)$, and the use of spirometry in horses was minimal. Non-invasive blood pressure was sometimes used in foals, when an arterial line had not been placed, or even with invasive blood pressure. These data are similar to those reported via the online questionnaire in 2015 by Wohlfender et al. [50].

The data show the current protocols for the induction of anaesthesia were usually assisted, with either personnel (61\%) or using a gate $(25 \%)$. For recovery, $51 \%$ were free, $41 \%$ were assisted with ropes and $8 \%$ were manually assisted, mostly foals. These data reflect the ongoing controversy about which is the best method of recovery from general anaesthesia. Head and tail rope systems do not completely prevent fractures during recovery [51-53], but some cases may benefit from this technique. Slings were used in some cases but again did not ensure a good recovery: one young horse became excited with sling assistance and recovered badly after an elbow fracture repair; this was one of the unexpected noncolic deaths.

(4) Preliminary results of standing sedations in horses

The data presented here are the first reported from a prospective, multicentre, cohort study of standing sedation, but the numbers are small and cautious interpretation is still in order.

Whereas the data collected from general anaesthetics can be considered as strong and reliable, only 43 of the 57 centres sent more than $90 \%$ of their standing sedation cases, which could lead to biased data. The main challenge for centres with a high case load was to collect every single case.

Alpha $_{2}$-agonists were used in all except three of the premedication combinations. Acepromazine was not given alone but, combined with at least an alpha $2_{2}$-agonist, it was given to $47 \%$ of the cases. Opioids, mostly butorphanol, morphine and methadone were used in $85 \%$ of the cases. Boluses of detomidine, butorphanol and romifidine are the most 
commonly used for the maintenance of standing sedations. For CRIs, detomidine was the most commonly used, followed by far, by butorphanol. Monitoring in standing sedation procedures was minimal.

Many of the procedures previously performed only in anaesthetised horses are now carried out using standing sedation. Although the inclusion criteria were more restrictive and not all the centres were able to supply these procedures, $23 \%$ of the cases in this study were standing sedations. Further study of these data is required. Creating a subgroup of collaborating centres may ensure more reliable data.

\section{(5) Limitations}

Our study is not free of limitations. First, the electronic questionnaire. Its use required a learning curve and sometimes raised technological difficulties, all solved with online support. The potential for creating a specific app is under consideration; however, the adaptation to different software can be challenging. Second, the current version of the questionnaire could be improved. For instance, the field "castrated" and "pregnant" is not mandatory in the current version and could lead to misinterpretation. Drugs commonly used, such as mepivacaine, were not included (but could be added manually). An updated version will be created in response to the feedback from the collaborating centres. Third, there is an inherent bias as some areas of the world and types of practice are underrepresented. Further strategies are to be implemented in this respect, although language barriers should not be underestimated. Finally, the amount of information collected by the questionnaire is enormous and cannot be covered in a single scientific paper. However, and as stated in the agreement form, "sub-studies can be proposed, as long as this does not involve duplicate use of the CEPEF4 data". This may allow our community to benefit from our method for collecting multicentric data for purposes other than mortality up to seven days.

\section{Conclusions}

We have designed a reliable method, with a clear strategy of communication and data collection/cleaning that can be used to collect cases for CEPEF4. This approach will help to minimize inconsistencies that may lead to data misinterpretation in future CEPEF studies. This preliminary report shows that horses still die unexpectedly during and within the seven-day postoperative period of general anaesthesia and standing sedation. Our results also show that current practice in anaesthesia has changed over the last 20 years.

Supplementary Materials: The following are available online at https:/ / www.mdpi.com/article/10 .3390/ani11092549/s1, S1: questionnaire and the instructions to complete it; S2: R scripts used in the data cleaning phase; S3: checklist of STROBE guidelines.

Author Contributions: Conceptualization, M.G.-M., R.B.-W., M.J., P.M.T. and J.I.R.; data curation, M.G.-M. and J.I.R.; formal analysis, J.I.R.; funding acquisition, M.G.-M. and R.B.-W.; investigation, M.G.-M., R.B.-W., M.J., P.M.T. and J.I.R.; methodology, M.G.-M., R.B.-W., M.J., P.M.T. and J.I.R.; project administration, M.G.-M.; resources, J.I.R.; software, J.I.R.; supervision, M.G.-M., R.B.-W., M.J., P.M.T. and J.I.R.; validation, M.G.-M., R.B.-W., M.J. and P.M.T.; writing-original draft, M.G.-M. and J.I.R.; writing-review and editing, M.G.-M., R.B.-W., M.J., P.M.T. and J.I.R. All authors have read and agreed to the published version of the manuscript.

Funding: The authors would like to thank the Kate Borer-Weir Fund of the Association of Veterinary Anaesthetists for supporting us in the initial phase of this study.

Institutional Review Board Statement: The study was approved by the international Ethical Review Committee of the Association of Veterinary Anaesthetists (AVA), under protocol 2020-009.

Informed Consent Statement: Not applicable. As in previous CEPEF studies, the anonymity and confidentiality of the patients, owners and centres was ensured.

Data Availability Statement: Raw data, converted to a .csv file, are stored in the CEPEF4 metadata file. The most relevant results can be accessed at: https:/ / cepef4.wordpress.com/preliminary-results / (accessed on 6 June 2021). 
Acknowledgments: The project would have been impossible without the open mindedness, hard work, honesty and diligence of the personnel of every single collaborating centre who submitted the cases for these preliminary results. The listed names of the ambassadors and the collaborating centres per country can be found in Appendix A. Special thanks also to Rob Ward (The University of Edinburgh, Edinburgh, UK) for his support in the design of the mortality survey for the noncolic deaths and Luis Domenech (Universidad CEU Cardenal Herrera, Valencia, Spain) for his IT support.

Conflicts of Interest: The authors declare no conflict of interest.

\section{Appendix A}

List of the collaborating centres and ambassadors per country, ordered alphabetically by country:

- Argentina: Centro de Cirugía Equina Santa Fe, Santa Fe (Natalia Rossetti); Hospital Escuela de Grandes Animales FCV UNNE, Corrientes (Eduardo Darío Saravia)

- Australia: Goulburn Valley Equine Hospital, Congupna, Victoria (Valentina Di Sevo); Scone Equine Hospital, Scone, New South Wales (Maria Helena Bellei)

- Austria: Veterinärmedizinische Universität Wien, Wien (Natalí Verdier)

- Belgium: Dierenkliniek de Bosdreef, Moerbeke-Waas, Oost-Vlaanderen (Katherine Bafort and Hans Wilderjans); Equitom Equine Clinic, Meldert, Limbürg (Jamien Deman and Tom Marien); Université de Liège, Liège, Wallonie (Julien Dupont and Charlotte Sandersen); Universiteit Gent, Merelbeke, Oost-Vlaanderen (Stijn Schauvliege and Frank Gasthuys)

- Brasil: Universidade Federal de Minas Gerais, Belo Horizonte, MG (Suzane Lilian Beier); Universidade Federal do Paraná, Curitiba, PR (Juan Carlos Duque Moreno); Universidade de São Paulo, Pirassununga, SP (Adriano Carregaro); Universidade de São Paulo, São Paulo, SP (Denise Tabacchi Fantoni)

- Canada: Université de Montréal, Saint Hyacinthe, Québec (Mathilde Ducroq, Javier Benito de la Víbora and Paulo Steagall); Western College of Veterinary Medicine, University of Saskatchewam, Saskatchewam (Barbara Ambros)

- Chile: HCV Universidad Nacional Andrés Bello, Santiago de Chile, Región Metropolitana (Karina Aros Villarroel)

- Colombia: Gustavo Villa Díaz (ambulatory clinician), Medellín, Antioquia; Universidad CES, Medellín, Antioquia (Gustavo Villa Díaz); Universidad Lasallista, Caldas, Antioquia (Gustavo Villa Díaz); Universidad Tecnológica de Pereira, Pereira, Risaralda (David Latorre Galeano)

- France: Centre Hospitalier Vétérinaire Équin de Livet, Calvados (Valerie Picanvet); Clinique de Grosbois, Boissy Saint Leger, Val de Marne (Valerie Deniau); Clinique Équine Acy Romance, Acy Romance, Grand Est (Justine Dalla Valle and Anneleen Michielsen); Clinique Équine de la Boisrie, Chaylloue, Orne (Peggy Moreau); Clinique Équine de Meslay, Pays de Loire (Bienvenida Román Durá and Tamara de Beauregard); Clinique Vétérinaire Équine de Meheudin, Écouche, Orne (Elsa Autard and Florence Polle)

- Germany: TIHO Hannover, Hannover, Niedersachsen (Lara Twele, Álvaro Gutiérrez Bautista and Sabine Kästner)

- Italy: Università di Bologna, Bologna (Carlotta Lambertini and Noemi Romagnoli); Università di Perugia, Perugia (Sara Nannarone)

- Japan: Shadai Horse Clinic, Hokkaido (Tsukasa Suzuki)

- Mexico: Central Quirúrgica para Caballos, Naucalpan, Estado de México (Jorge Eduardo Mendoza Flores); EQCOVET, Zapopan, Jalisco (Edgardo Hernández Díaz); Jorge Eduardo Mendoza Flores (ambulatory clinician), Ciudad de México, Estado de México

- Republic of Ireland: Fethard Equine Hospital, Clonmel, Tipperary (Erica Sogus); Somerton Equine Hospital, Friarstown, Kildare (Monica McNichol and Valerie Hyde); University College Dublin, Dublin, Dublin (Vilhelmiina Huuskonen)

- Spain: Hospital de Referencia La Equina, Manilva, Málaga (Joana Maia Ramos, Maya Caparrós Buscarons and Miguel Valdés Vázquez); Hospital Veteriario Costa de La Luz, Conil de la Frontera, Cádiz (Ana del Ojo Morales); Universidad Alfonso X 
El Sabio, Villanueva de la Cañada, Madrid (Rocío Cañellas Blanco and Verónica Salazar Nussio); Universidad Autónoma de Barcelona, Barcelona (Lucas Troya Portillo and Cristina Costa Farré); Universidad Cardenal Herrera CEU, Valencia (Eva Zoe Hernández Magaña, Jaime Viscasillas Monteagudo and José Ignacio Redondo García); Universidad de Córdoba, Córdoba (Esther Caravaca Paredes and María del Mar Granados Machuca); Universidad Complutense de Madrid, Madrid (Marta Villalba Díez, Isabel Santiago Llorente and Ignacio Álvarez Gómez de Segura); Universidad de Murcia, Murcia (Eliseo Belda Mellado and Francisco Ginés Laredo Álvarez)

- Switzerland: Bern Universität, Bern (Ilaria Petruccione and Olivier Levionnois); Pferdeklinik Dalchenhof, Brittnau, Aargau (Orquidea Kiesse); Pferdeklinik Niederlenz, Niederlenz, Lenzburg (Theo Tschanz); Vetsuisse Faculty, University of Zürich, Zürich (Simone Katja Ringer and Regula Bettschart-Wolfensberger)

- The Netherlands: Dierenhospital Visdonk, Roosendaal (Els van den Berg); Universiteit Utrecht, Utrecht (Janny de Grauw and Thijs van Loon)

- United Kingdom: Ashbrook Equine Hospital, Allostock, Cheshire (Julia Shrubb); Bell

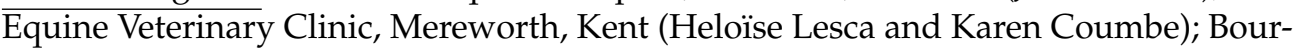
ton Vale Equine Clinic, Cheltenham, Gloucestershire (Lydia Coles); Hird \& Partners, Shelf, West Yorkshire (John Hird); Liphook Equine Hospital, Liphook, Hampshire (Rachel Tucker); Pool House Equine Clinic, Fradley, Staffordshire (Federica Cantatore, Cristina Bertone and Sophie Malorey); Rainbow Equine Hospital, Malton, North Yorkshire (Kate Loomes); Rossdales Ltd., Newmarket, Suffolk (Luis Louro and Catriona McKenzie); Royal Veterinary College, Hatfield, Hertfordshire (Kata Veres-Nyeki); The Donkey Sanctuary, Sidmouth, Devon (Abigail Sefton, Dom Doyle and Rebekah Sullivan); The Universisty of Edinburgh, Easter Bush Campus, Midlothian (Chiara Pressanto, Tom Beeston and Miguel Gozalo-Marcilla); University of Glasgow, Bearsden, Glasgow (Josephine Kropf and Andrew Bell); Wright and Morten, Somerford, Cheshire (Nicola Harries and Rachel Helm)

- United States of America: Colorado State University, Fort Collins, CO (Rachel Hector and Marlis Rezende); University of Missouri, Columbia, MO (Kelley Varner); University of Pennsilvania-New Bolton Centre, Kennett Square, PA (Klaus Hopster)

- Uruguay: Facultad de Veterinaria-UDELAR, Montevideo (Sabrina Castro Peri, Gastón Pérez and Camila Nan); María Noel Acevedo (ambulatory clinician), Montevideo; Servicio de Veterinaria y Remonta, Canelones (Sabrina Castro Peri and María Noel Acevedo)

Also, we would like to thank our colleagues that provided us the contacts of clinics in the following countries/continents:

- Belgium (Stijn Schauvliege)

- France (Latifa Khenissi and Stijn Schauvliege)

- Japan (Paulo Steagall)

- México (José Antonio Ibancovichi)

- South America (Adriano Carregaro and Natalia Rossetti)

- The Netherlands (Janny de Grauw) 


\section{References}

1. Ahern, N.R. Using the Internet to conduct research. Nurse Res. 2005, 13, 55-70. [CrossRef] [PubMed]

2. Cantrell, M.A.; Lupinacci, P. Methodological issues in online data collection. J. Adv. Nurs. 2007, 60, 544-549. [CrossRef] [PubMed]

3. Pace, W.D.; Staton, E.W. Electronic data collection options for practice-based research networks. Ann. Fam. Med. 2005, 3 (Suppl. S1), S21-S29. [CrossRef] [PubMed]

4. Pittard, W.S.; Li, S. The essential toolbox of data science: Python, R, Git, and Docker. Methods Mol. Biol. 2020, $2104,265-311$. [CrossRef] [PubMed]

5. Van den Broeck, J.; Cunningham, S.A.; Eeckels, R.; Herbst, K. Data cleaning: Detecting, diagnosing, and editing data abnormalities. PLoS Med. 2005, 2, e267. [CrossRef]

6. Mitchell, B. Equine anaesthesia: An assessment of techniques used in clinical practice. Equine Vet. J. 1969, 1, 261-275. [CrossRef]

7. Tevik, A. The role of anesthesia in surgical mortality in horses. Nord. Vet. Med. 1983, 35, 175-179.

8. Young, S.S.; Taylor, P.M. Factors influencing the outcome of equine anaesthesia: A review of 1,314 cases. Equine Vet. J. 1993, 25, 147-151. [CrossRef]

9. Mee, A.M.; Cripps, P.J.; Jones, R.S. A retrospective study of mortality associated with general anaesthesia in horses: Elective procedures. Vet. Rec. 1998, 142, 275-276. [CrossRef]

10. Mee, A.M.; Cripps, P.J.; Jones, R.S. A retrospective study of mortality associated with general anaesthesia in horses: Emergency procedures. Vet. Rec. 1998, 142, 307-309. [CrossRef]

11. Bidwell, L.A.; Bramlage, L.R.; Rood, W.A. Equine perioperative fatalities associated with general anaesthesia at a private practice-a retrospective case series. Vet. Anaesth. Analg. 2007, 34, 23-30. [CrossRef] [PubMed]

12. Jago, R.C.; Corletto, F.; Wright, I.M. Peri-anaesthetic complications in an equine referral hospital: Risk factors for post anaesthetic colic. Equine Vet. J. 2015, 47, 635-640. [CrossRef] [PubMed]

13. Dugdale, A.H.; Obhrai, J.; Cripps, P.J. Twenty years later: A single-centre, repeat retrospective analysis of equine perioperative mortality and investigation of recovery quality. Vet. Anaesth. Analg. 2016, 43, 171-178. [CrossRef] [PubMed]

14. Laurenza, C.; Ansart, L.; Portier, K. Risk factors of anesthesia-related mortality and morbidity in one equine hospital: A retrospective study on 1,161 cases undergoing elective or emergency surgeries. Front. Vet. Sci. 2020, 6, 514. [CrossRef] [PubMed]

15. Proudman, C.J.; Dugdale, A.H.; Senior, J.M.; Edwards, G.B.; Smith, J.E.; Leuwer, M.L.; French, N.P. Pre-operative and anaesthesiarelated risk factors for mortality in equine colic cases. Vet. J. 2006, 171, 89-97. [CrossRef] [PubMed]

16. Johnston, G.M.; Taylor, P.M.; Holmes, M.A.; Wood, J.L. Confidential enquiry of perioperative equine fatalities (CEPEF-1): Preliminary results. Equine Vet. J. 1995, 27, 193-200. [CrossRef] [PubMed]

17. Senior, J.M.; Pinchbeck, G.L.; Allister, R.; Dugdale, A.H.; Clark, L.; Clutton, R.E.; Coumbe, K.; Dyson, S.; Clegg, P.D. Reported morbidities following 861 anaesthetics given at four equine hospitals. Vet. Rec. 2007, 160, 407-408. [CrossRef]

18. Johnston, G.M.; Eastment, J.K.; Wood, J.; Taylor, P.M. The confidential enquiry into perioperative equine fatalities (CEPEF) Mortality results of Phases 1 and 2. Vet. Anaesth. Analg. 2002, 29, 159-170. [CrossRef]

19. Dugdale, A.H.; Taylor, P.M. Equine anaesthesia-associated mortality: Where are we now? Vet. Anaesth. Analg. 2016, 43, $242-255$. [CrossRef]

20. Gent, T.C.; Bettschart-Wolfensberger, R. Peri-anaesthetic mortality in horses-the need for CEPEF-4. Vet. Anaesth. Analg. 2013, 40, e1-e2. [CrossRef]

21. Redondo, J.I. Anaesthetic-related deaths in dogs in Spain: COMPLRED 2016-2019. In Proceedings of the Autumn Meeting of the Association of Veterinary Anaesthetists 2019, Ghent, Belgium, 11-13 September 2019; p. 166. Available online: https: / / ava.eu.com/wp-content/uploads/2019/09/Proceedings-Ghent-2019-main-congress.pdf (accessed on 6 June 2021).

22. Gozalo-Marcilla, M.; Redondo, J.I.; Johnston, M.; Taylor, P.; Bettschart-Wolfensberger, R. CEPEF4: Update and plan. Vet. Anaesth. Analg. 2020, 47, 724-725. [CrossRef]

23. Gozalo-Marcilla, M.; Redondo, J.I.; Johnston, M.; Taylor, P.M.; Bettschart-Wolfensberger, R. CEPEF4 going live. In Proceedings of the Spring Meeting of the Association of Veterinary Anaesthetists 2020, Dublin, Ireland, 11-13 March 2020; p. 90. Available online: http:/ / avadublin2020.ie/wp-content/uploads/2020/03/FINAL-AVA-Proceedings.pdf (accessed on 6 June 2021).

24. Gozalo-Marcilla, M.; Redondo, J.I.; Johnston, M.; Taylor, P.; Bettschart-Wolfensberger, R. A new equine anaesthetic mortality study two decades after CEPEF2: CEPEF4 is going live! Equine Vet. J. 2020, 52, 891-892. [CrossRef]

25. R Core Team. R: A Language and Environment for Statistical Computing; R Foundation for Statistical Computing: Vienna, Austria, 2021; Available online: https: / /www.R-project.org/ (accessed on 6 June 2021).

26. Rich, B. Table 1: Tables of Descriptive Statistics in HTML. 2021, R Package Version 1.4.1. Available online: https:/ CRAN.Rproject.org / package=table1 (accessed on 6 June 2021).

27. Wickham, H. ggplot2: Elegant Graphics for Data Analysis; Springer: New York, NY, USA, 2016.

28. South, A. Rnaturalearth: World Map Data from Natural Earth. R Package Version 0.1.0. 2017. Available online: https: / /CRAN.R-project.org/package=rnaturalearth (accessed on 6 June 2021).

29. von Elm, E.; Altman, D.G.; Egger, M.; Pocock, S.J.; Gøtzsche, P.C.; Vandenbroucke, J.P.; STROBE Initiative. The Strengthening the Reporting of Observational Studies in Epidemiology (STROBE) Statement: Guidelines for reporting observational studies. Int. J. Surg. 2014, 12, 1495-1499. [CrossRef] [PubMed]

30. Brodbelt, D.C.; Blissitt, K.J.; Hammond, R.A.; Neath, P.J.; Young, L.E.; Pfeiffer, D.U.; Wood, J.L. The risk of death: The confidential enquiry into perioperative small animal fatalities. Vet. Anaesth. Analg. 2008, 35, 365-373. [CrossRef] [PubMed] 
31. Clarke, K.W.; Paton, B.S. Combined use of detomidine with opiates in the horse. Equine Vet. J. 1988, 20, 331-334. [CrossRef] [PubMed]

32. Lopes, C.; Luna, S.P.; Rosa, A.C.; Quarterone, C.; Crosignani, N.; Taylor, P.M.; Pantoja, J.C.; Puoli, J.N. Antinociceptive effects of methadone combined with detomidine or acepromazine in horses. Equine Vet. J. 2016, 48, 613-618. [CrossRef]

33. Gozalo-Marcilla, M.; Luna, S.P.; Crosignani, N.; Filho, J.N.P.; Possebon, F.S.; Pelligand, L.; Taylor, P.M. Sedative and antinociceptive effects of different combinations of detomidine and methadone in standing horses. Vet. Anaesth. Analg. 2017, 44, 1116-1127. [CrossRef] [PubMed]

34. Hall, L.W. Equine anaesthesia: Discovery and rediscovery. Equine Vet. J. 1983, 15, 190-195. [CrossRef] [PubMed]

35. Taylor, P.M. The stress response to anaesthesia in ponies: Barbiturate anaesthesia. Equine Vet. J. 1990, 22, 307-312. [CrossRef]

36. Posner, L.P.; Kasten, J.I.; Kata, C. Propofol with ketamine following sedation with xylazine for routine induction of general anaesthesia in horses. Vet. Rec. 2013, 173, 550. [CrossRef]

37. Johnston, G.M.; Eastment, J.K.; Taylor, P.M.; Wood, J.L. Is isoflurane safer than halothane in equine anaesthesia? Results from a prospective multicentre randomised controlled trial. Equine Vet. J. 2004, 36, 64-71. [CrossRef] [PubMed]

38. Gozalo-Marcilla, M.; Gasthuys, F.; Schauvliege, S. Partial intravenous anaesthesia in the horse: A review of intravenous agents used to supplement equine inhalation anaesthesia. Part 1: Lidocaine and ketamine. Vet. Anaesth. Analg. 2014, 41, 335-345. [CrossRef] [PubMed]

39. Gozalo-Marcilla, M.; Gasthuys, F.; Schauvliege, S. Partial intravenous anaesthesia in the horse: A review of intravenous agents used to supplement equine inhalation anaesthesia. Part 2: Opioids and alpha-2 adrenoceptor agonists. Vet. Anaesth. Analg. 2015, 42, 1-16. [CrossRef] [PubMed]

40. White, K. Total and partial intravenous anaesthesia of horses. Practice 2015, 37, 189-197. [CrossRef]

41. Devisscher, L.; Schauvliege, S.; Dewulf, J.; Gasthuys, F. Romifidine as a constant rate infusion in isoflurane anaesthetized horses: A clinical study. Vet. Anaesth. Analg. 2010, 37, 425-433. [CrossRef] [PubMed]

42. Sacks, M.; Ringer, S.K.; Bischofberger, A.S.; Berchtold, S.M.; Bettschart-Wolfensberger, R. Clinical comparison of dexmedetomidine and medetomidine for isoflurane balanced anaesthesia in horses. Vet. Anaesth. Analg. 2017, 44, 1128-1138. [CrossRef]

43. Valverde, A.; Gunkelt, C.; Doherty, T.J.; Giguère, S.; Pollak, A.S. Effect of a constant rate infusion of lidocaine on the quality of recovery from sevoflurane or isoflurane general anaesthesia in horses. Equine Vet. J. 2005, 37, 559-564. [CrossRef]

44. Larenza, M.P.; Peterbauer, C.; Landoni, M.F.; Levionnois, O.L.; Schatzmann, U.; Spadavecchia, C.; Thormann, W. Stereoselective pharmacokinetics of ketamine and norketamine after constant rate infusion of a subanesthetic dose of racemic ketamine or S-ketamine in Shetland ponies. Am. J. Vet. Res. 2009, 70, 831-839. [CrossRef]

45. Chesnel, M.A.; Clutton, R.E. A comparison of two morphine doses on the quality of recovery from general anaesthesia in horses. Res. Vet. Sci. 2013, 95, 1195-1200. [CrossRef]

46. Gozalo-Marcilla, M.; Steblaj, B.; Schauvliege, S.; Duchateau, L.; Gasthuys, F. Comparison of the influence of two different constantrate infusions (dexmedetomidine versus morphine) on anaesthetic requirements, cardiopulmonary function and recovery quality in isoflurane anaesthetized horses. Res. Vet. Sci. 2013, 95, 1186-1194. [CrossRef]

47. Santos, M.; Fuente, M.; Garcia-Iturralde, R.; Herran, R.; Lopez-Sanroman, J.; Tendillo, F.J. Effects of alpha-2 adrenoceptor agonists during recovery from isoflurane anaesthesia in horses. Equine Vet. J. 2003, 35, 170-175. [CrossRef]

48. Woodhouse, K.J.; Brosnan, R.J.; Nguyen, K.Q.; Moniz, G.W.; Galuppo, L.D. Effects of postanesthetic sedation with romifidine or xylazine on quality of recovery from isoflurane anesthesia in horses. J. Am. Vet. Med. Assoc. 2013, 242, 533-539. [CrossRef]

49. Guedes, A.G.P.; Tearney, C.C.; Cenani, A.; Aristizabal, F.; Nieto, J. Comparison between the effects of postanesthetic xylazine and dexmedetomidine on characteristics of recovery from sevoflurane anesthesia in horses. Vet. Anaesth. Analg. 2017, 44, 273-280. [CrossRef] [PubMed]

50. Wohlfender, F.D.; Doherr, M.G.; Driessen, B.; Hartnack, S.; Johnston, G.M.; Bettschart-Wolfensberger, R. International online survey to assess current practice in equine anaesthesia. Equine Vet. J. 2015, 47, 65-71. [CrossRef] [PubMed]

51. Arndt, S.; Hopster, K.; Sill, V.; Rohn, K.; Kastner, S.B.R. Comparison between head-tail-rope assisted and unassisted recoveries in healthy horses undergoing general anesthesia for elective surgeries. Vet. Surg. 2020, 49, 329-338. [CrossRef] [PubMed]

52. Ruegg, M.; Bettschart-Wolfensberger, R.; Hartnack, S.; Junge, H.K.; Theiss, F.; Ringer, S.K. Comparison of non-assisted versus head and tail rope-assisted recovery after emergency abdominal surgery in horses. Pferdeheilkunde 2016, 32, 469-478. [CrossRef]

53. del Barrio, M.C.N.; David, F.; Hughes, J.M.L.; Clifford, D.; Wilderjans, H.; Bennett, R. A retrospective report (2003-2013) of the complications associated with the use of a one-man (head and tail) rope recovery system in horses following general anaesthesia. Ir. Vet. J. 2018, 71, 6. [CrossRef] 